\title{
Eight dimensional QCD at one loop
}

\author{
J. A. Gracey \\ Theoretical Physics Division, Department of Mathematical Sciences, University of Liverpool, \\ P.O. Box 147, Liverpool, L69 3BX, United Kingdom
}

(Received 31 August 2017; published 16 January 2018)

\begin{abstract}
The Lagrangian for a non-Abelian gauge theory with an $S U\left(N_{c}\right)$ symmetry and a linear covariant gauge fixing is constructed in eight dimensions. The renormalization group functions are computed at one loop with the special cases of $N_{c}=2$ and 3 treated separately. By computing the critical exponents derived from these in the large $N_{f}$ expansion at the Wilson-Fisher fixed point it is shown that the Lagrangian is in the same universality class as the two dimensional non-Abelian Thirring model and quantum chromodynamics (QCD). As the eight dimensional Lagrangian contains new quartic gluon operators not present in four dimensional QCD, we compute in parallel the mixing matrix of four dimensional dimension 8 operators in pure Yang-Mills theory.
\end{abstract}

DOI: 10.1103/PhysRevD.97.025009

\section{INTRODUCTION}

Non-Abelian gauge theories are established as the core quantum field theories which govern the particles of nature through the Standard Model. One sector, which is known as quantum chromodynamics (QCD), describes the strong force between fundamental quarks and gluons which leads to the binding of these quanta into the mesons and hadrons seen in Nature. QCD has rather distinct properties in comparison with the electroweak sector. For instance, at high energy quarks and gluons become effectively free particles due to the property of asymptotic freedom, [1,2]. While this attribute is essential to developing a field theoretic formalism which allows us to extract meaningful information from experimental data, it has an implicit sense that at lower energies quarks and gluons can never be treated as distinct particles in the same spirit as a free electron in quantum electrodynamics (QED) which is an Abelian gauge theory. The concept of a lack of low energy freedom is known as colour confinement or infrared slavery in contradistinction to the virtual freedom at ultraviolet scales. As it stands QCD has been studied in depth over many years. One area where there has been significant progress recently is in the evaluation of the fundamental renormalization group functions at very high loop order. For instance, following the one loop discovery of asymptotic freedom, [1,2], the two and three loop corrections to the $\beta$-function appeared within a decade [3-5]. Progress to

Published by the American Physical Society under the terms of the Creative Commons Attribution 4.0 International license. Further distribution of this work must maintain attribution to the author(s) and the published article's title, journal citation, and DOI. Funded by SCOAP ${ }^{3}$. the four loop term followed in the 1990s, [6,7], before a lull to the recent five loop explosion of all the renormalization group functions [8-15]. By this we mean the $\beta$-function was determined for the $S U(3)$ color group in [9] before this was extended to a general Lie group in [10]. The supporting five loop renormalization group functions were determined in [8,11-15]. While such multiloop QCD results are impressive in the extreme, in the overall scheme of things having independent checks on such calculations is useful. The recent five loop QCD $\beta$-function of [9] is relatively unique in this respect in that the independent computation of [10] followed quickly. Ordinarily such a task requires as much human and computer resources as the initial breakthrough which are not always immediately available.

For QCD there is a parallel method of verifying part of the perturbative series which is via the large $N_{f}$ expansion where $N_{f}$ is the number of massless quarks. For instance, the QCD $\beta$-function was determined at $O\left(1 / N_{f}\right)$ in [16] which extended the QED result of [17]. Subsequently the quark mass anomalous dimension was found at $O\left(1 / N_{f}^{2}\right)$ in [18]. The $1 / N_{f}$ or large $N_{f}$ expansion provides an alternative way of deducing certain coefficients in the perturbative series and the work of $[16,18]$ extended the original method for spin- 0 fields of $[19,20]$ to the spin- 1 case. However, the formalism for the gauge theory context derives from a novel and elegant observation made in [21]. In [21] it was shown that the non-Abelian Thirring model (NATM) in the large $N_{f}$ expansion is in the same universality class as QCD at the Wilson-Fisher fixed point in $d$-dimensions. While the non-Abelian Thirring model is a nonrenormalizable quantum field theory above two dimensions, within the large $N_{f}$ expansion at its $d$-dimensional fixed point the $d$-dimensional critical exponents 
contain information on the perturbative renormalization group functions of QCD. This has been verified by agreement with the latest set of five loop renormalization group functions [8-15]. The novel feature is the fact that in the non-Abelian Thirring model there are no triple and quartic gluon self-interactions as is well known in QCD. These vertices effectively emerge at criticality within large $N_{f}$ computations via 3- and 4-point quark loops, [21]. More recently this property of critical equivalence has been studied in the simpler $O(N)$ scalar field theories where a similar phenomenon of higher dimensional theory vertices are generated at criticality by triangle and box graphs. In more modern parlance this is known as ultraviolet completion. Indeed in the $O(N)$ nonlinear $\sigma$ model and $O(N) \phi^{4}$ theory, the Wilson-Fisher fixed point equivalence in $2<d<4$ was extended to six dimensional $O(N) \phi^{3}$ theory in [22,23] and then beyond in [24,25].

In light of this the six dimensional extension of the nonAbelian Thirring model and QCD equivalence was provided in [26]. This involved a more intricate Lagrangian but the connection of the two loop renormalization group functions with the universal $d$-dimensional large $N_{f}$ critical exponents was verified. Again this reinforced the remarkable connection with the non-Abelian Thirring model in that the six dimensional theory has quintic and sextic gluon selfinteractions in addition to cubic and quartic structures which are the only ones present in four dimensions. While formally there are cubic and quartic interactions in both these dimensions, the Feynman rules of the vertices are different in each dimension. So the fact that the large $N_{f}$ non-Abelian Thirring model exponents encode information on the respective renormalization group functions is remarkable since it is not a gauge theory as such. Given this background it is therefore the purpose of this article to continue the tower of theories to the next link in the chain and construct the eight dimensional non-Abelian theory in what we will now term the non-Abelian Thirring model universality class. This runs parallel to the six and eight dimensional extensions of QED $[26,27]$. The eight dimensional non-Abelian theory has significantly more structure in its Lagrangian. For instance, there are seven independent quartic field strength operators in general as opposed to two in the QED case [26]. Equally one has a higher power propagator for the gluon and Faddeev-Popov ghost fields which means evaluating Feynman integrals even at one loop becomes a significant task. Therefore in this article we concentrate on a full one loop renormalization of the field anomalous dimensions and all the $\beta$-functions. As such one can regard this as proof of concept to launch a two loop computation from. The eight dimensional QED evaluation of [26] was able to probe to two loops partly because of fewer interactions but also as a consequence of the Ward-Takahashi identity.

A parallel reason for examining six and eight dimensional gauge theories rests in the connection to operators in lower dimensions. If one has the viewpoint of an underlying universal theory residing at a fixed point in $d$ dimensions, then the gauge independent operators corresponding to the interactions of the higher dimensional theory have dimensionless coupling constants in their respective critical dimensions. Below this dimension the coupling constant would become massive. Therefore they would equate to operators in the effective field theory of the lower dimensional gauge theory. In [26] it was noted that in the six dimensional extension of QCD the fully massive gluon propagator in the Landau gauge bore a remarkable qualitative similarity to the infrared behavior of the propagator as computed in the same gauge on the lattice but in four dimensions. While there was an observation in $[28,29]$ that the ultraviolet behavior of a higher dimensional theory informs or models the infrared structure of a lower dimensional one, it would seem that an eight dimensional one could only relate to infrared fixed points in its six dimensional partner. However, given that dimension 8 operators are of interest in four dimensional effective field theories of QCD having renormalization group function data in the eight dimensional non-Abelian gauge theory for $S U\left(N_{c}\right)$, where $N_{c}$ is the number of colors, is an additional motivation for future studies. In four dimensions such dimension 8 operators were studied in [29] for Yang-Mills theories for the $S U(2)$ and $S U(3)$ color groups. Here we extend the set and provide the one loop mixing matrix of dimension 8 operators in four dimensional $S U\left(N_{c}\right)$ Yang-Mills theory. It will turn out that there are qualitative structural similarities between the matrix and the $\beta$-functions of the eight dimensional theory.

The article is organized as follows. We discuss the construction of the eight dimensional Lagrangian which will be in the same universality class as the non-Abelian Thirring model and QCD in the next section. The technology used to renormalize the various $n$-point functions in this Lagrangian is discussed in Sec. III before presenting the main results in Sec. IV. The connection with the large $N_{f}$ expansion of the critical exponents of the universality class is checked in Sec. V. In Sec. VI we change tack and determine the mixing matrix of anomalous dimensions of dimension 8 operators in four dimensional Yang-Mills theory. Finally, concluding remarks are given in Sec. VII.

\section{BACKGROUND}

As the first stage to constructing the eight dimensional version of QCD we recall the corresponding Lagrangians of the lower dimensional cases. The four dimensional Lagrangian is

$$
L^{(4)}=-\frac{1}{4} G_{\mu \nu}^{a} G^{a \mu \nu}+i \bar{\psi}^{i I} \not D \psi^{i I}-\frac{1}{2 \alpha}\left(\partial^{\mu} A_{\mu}^{a}\right)^{2}-\bar{c}^{a}\left(\partial^{\mu} D_{\mu} c\right)^{a}
$$

where we have included the canonical linear covariant gauge fixing term with the associated Faddeev-Popov ghost. In (2.1) and throughout the gluon field will be 
denoted by $A_{\mu}^{a}$, the quark field will be $\psi^{i I}$ and $c^{a}$ are the Faddeev-Popov ghost fields where $1 \leq i \leq N_{f}, 1 \leq I \leq N_{F}$ and $1 \leq a \leq N_{A}$. The parameters $N_{f}, N_{A}$ and $N_{F}$ correspond respectively to the number of (massless) quark flavors and the dimensions of the adjoint and fundamental representations of a general color group. We use $\alpha$ as the linear covariant gauge parameter where $\alpha=0$ will correspond to the Landau gauge. To assist with the process of writing down the Lagrangians which are equivalent to (2.1) in higher dimensions one can regard (2.1) as being comprised of two parts. The first is the set of independent gauge invariant operators of dimension four built from the gluon and quark fields which have canonical dimensions of 1 and $\frac{3}{2}$ in four dimensions. Then in order to be able to carry out explicit computations in perturbation theory, for instance, one has to add in the appropriate gauge fixing term to ensure that a nonsingular propagator can be constructed for the gluon. This is the gauge fixing part of (2.1). From an operator point of view this involves the independent gauge variant dimension four operators. By independent we mean those operators which are not related by linear combinations of total derivative operators. Given this the six dimensional extension of (2.1) was provided in [24] based on similar work given in [30]. With the increase in dimension the canonical dimension of the quark field is now $\frac{5}{2}$ which means that there are no quartic quark interactions. However, there are two independent gauge invariant gluonic operators which are apparent in the Lagrangian [24],

$$
\begin{aligned}
L^{(6)}= & -\frac{1}{4}\left(D_{\mu} G_{\nu \sigma}^{a}\right)\left(D^{\mu} G^{a \nu \sigma}\right)+\frac{g_{2}}{6} f^{a b c} G_{\mu \nu}^{a} G^{b \mu \sigma} G^{c \nu}{ }_{\sigma} \\
& -\frac{1}{2 \alpha}\left(\partial_{\mu} \partial^{\nu} A_{\nu}^{a}\right)\left(\partial^{\mu} \partial^{\sigma} A_{\sigma}^{a}\right)-\bar{c}^{a} \square\left(\partial^{\mu} D_{\mu} c\right)^{a} \\
& +i \bar{\psi}^{i I} \not D \psi^{i I}
\end{aligned}
$$

which means that there are two coupling constants. Demonstrating the independence of the gluonic operators lies in part with the use of the Bianchi identity

$$
D_{\mu} G_{\nu \sigma}^{a}+D_{\nu} G_{\sigma \mu}^{a}+D_{\sigma} G_{\mu \nu}^{a}=0 .
$$

The remaining gauge invariant operator is the quark kinetic term wherein lies the quark-gluon interaction which is the core interaction in the tower of theories at the Wilson-Fisher fixed point. Throughout we will always denote the usual gauge coupling constant by $g_{1}$ when there are one or more interactions. The remaining part of (2.2) is completed with the dimension six linear covariant gauge fixing term which is the obvious extension of the four dimensional one.

Equipped with this brief review of the construction of the dimension four and six non-Abelian gauge theories, the algorithm is now in place to proceed to eight dimensions. In $[31,32]$ the renormalization of dimension eight operators in four dimensional Yang-Mills theory was considered and those articles serve as the basis for the eight dimensional Lagrangian. As was discussed in [31] there is only one independent dimension eight 2-point gauge invariant operator which therefore serves as the gluon kinetic term. Equally $[31,32]$ there are two independent dimension eight 3 -point gluon operators. The new feature in eight dimensions, which derives from the fact that the gluon canonical dimension is unity, is that there will be quartic gluon field strength gauge invariant operators. The same property is present in eight dimensional QED which was introduced in [26] where there were several quartic photon selfinteractions. For the non-Abelian case there is the added complication of having to incorporate the color group indices. The upshot is that one has to specify a particular color group as it is not possible to have a finite set of quartic gluon opertors for a general Lie group [31]. Therefore we restrict ourselves to the $S U\left(N_{c}\right)$ Lie group and recall relevant basic properties of this group needed for the Lagrangian. If $T^{a}$ is the Lie group generator then in $S U\left(N_{c}\right)$ the product of two generators can be written as the linear combination

$$
T^{a} T^{b}=\frac{1}{2 N_{c}} \delta^{a b}+\frac{1}{2} d^{a b c} T^{c}+\frac{i}{2} f^{a b c} T^{c}
$$

where $d^{a b c}$ is totally symmetric and the structure constants, $f^{a b c}$, are totally antisymmetric. Equally when we have to treat Feynman graphs with quarks, the $S U\left(N_{c}\right)$ relation

$$
T_{I J}^{a} T_{K L}^{a}=\frac{1}{2}\left[\delta_{I L} \delta_{K J}-\frac{1}{N_{c}} \delta_{I J} \delta_{K L}\right]
$$

will be useful. To define gauge independent quartic gluon operators we introduce the rank 4 color tensors

$$
f_{4}^{a b c d} \equiv f^{a b e} f^{c d e}, \quad d_{4}^{a b c d} \equiv d^{a b e} d^{c d e}
$$

and then use the $S U\left(N_{c}\right)$ relation between them [33],

$$
f_{4}^{a b c d}=\frac{2}{N_{c}}\left(\delta^{a c} \delta^{b d}-\delta^{a d} \delta^{b c}\right)+d_{4}^{a c b d}-d_{4}^{a d b c} .
$$

This in effect [33] is the generalization of the relation between the product of Levi-Civita tensors in $S U(2)$ to the color groups $S U\left(N_{c}\right)$ for $N_{c} \geq 3$. It means that we use the tensor $d_{4}^{a b c d}$ as the preferred tensor of the gauge invariant operators. One reason for this is that $d_{4}^{a b c d}$ is separately symmetric in the first or last pair of indices from the full symmetry property of $d^{a b c}$. Consequently there are eight gauge independent quartic gluon operators in the eight dimensional extension of the QCD Lagrangian leading to eleven independent coupling constants overall. The full Lagrangian is 


$$
\begin{aligned}
L^{(8)}= & -\frac{1}{4}\left(D_{\mu} D_{\nu} G_{\sigma \rho}^{a}\right)\left(D^{\mu} D^{\nu} G^{a \sigma \rho}\right)+\frac{g_{2}}{4} f^{a b c} G_{\mu \nu}^{a} D^{\mu} G^{b \sigma \rho} D^{\nu} G_{\sigma \rho}^{c}+i \bar{\psi}^{i I} \not D \psi^{i I}+\frac{g_{3}}{2} f^{a b c} G_{\mu \nu}^{a} D_{\sigma} G^{b \mu \rho} D^{\sigma} G^{c \nu}{ }_{\rho} \\
& +g_{4}^{2} G_{\mu \sigma}^{a} G^{a \mu \rho} G^{b \sigma \nu} G_{\rho \nu}^{b}+g_{5}^{2} G_{\mu \sigma}^{a} G^{b \mu \rho} G^{b \sigma \nu} G_{\rho \nu}^{a}+g_{6}^{2} G_{\mu \sigma}^{a} G_{\nu \rho}^{a} G^{b \sigma \mu} G^{b \rho \nu}+g_{7}^{2} G_{\mu \sigma}^{a} G_{\nu \rho}^{b} G^{a \sigma \mu} G^{b \rho \nu}+g_{8}^{2} d_{4}^{a b c d} G_{\mu \sigma}^{a} G^{b \mu \sigma} G_{\nu \rho}^{c} G^{d \nu \rho} \\
& +g_{9}^{2} d_{4}^{a b c d} G_{\mu \sigma}^{a} G^{c \mu \rho} G^{b \nu \sigma} G_{\nu \rho}^{d}+g_{10}^{2} d_{4}^{a c b d} G_{\mu \sigma}^{a} G^{b \mu \sigma} G_{\nu \rho}^{c} G^{d \nu \rho}+g_{11}^{2} d_{4}^{a d b c} G_{\mu \sigma}^{a} G^{c \mu \rho} G^{b \nu \sigma} G_{\nu \rho}^{d}-\frac{1}{2 \alpha}\left(\partial_{\mu} \partial_{\nu} \partial^{\sigma} A_{\sigma}^{a}\right)\left(\partial^{\mu} \partial^{\nu} \partial^{\rho} A_{\rho}^{a}\right) \\
& -\left(\square \bar{c}^{a}\right)\left(\square \partial^{\mu} D_{\mu} c\right)^{a}
\end{aligned}
$$

where like (2.1) and (2.2) the dimension eight linear covariant gauge fixing term is included. In addition the quark kinetic term is present and is equivalent to those in the lower dimensional Lagrangians which therefore preserves the connection with the Wilson-Fisher fixed point and the underlying universal theory which is accessible from the large $N_{f}$ expansion. While (2.8) represents the full $S U\left(N_{c}\right)$ Lagrangian those for $N_{c}=2$ and 3 are smaller due to properties of the color tensors. For instance, for the $S U$ (2) group $d^{a b c}=0$. So for that group one has $g_{8}=$ $g_{9}=g_{10}=g_{11}=0$. For $S U(3) d^{a b c} \neq 0$ but $d_{4}^{a b c d}$ satisfies

$d_{4}^{a d b c}=-d_{4}^{a b c d}-d_{4}^{a c b d}+\frac{1}{3}\left[\delta^{a b} \delta^{c d}+\delta^{a c} \delta^{b d}+\delta^{a d} \delta^{b c}\right]$.

This means that two of the operators involving $d_{4}^{a b c d}$ are absent and within our computations we have set $g_{10}=$ $g_{11}=0$ for $S U(3)$. Finally we note several useful $S U\left(N_{c}\right)$ group identities, which we used within our graph evaluations, that are [33]

$$
\begin{aligned}
d_{4}^{a b c c} & =0, \quad d_{4}^{a c b c}=\frac{\left[N_{c}^{2}-4\right]}{N_{c}} \delta^{a b}, \\
d_{4}^{a p b q} d_{4}^{c d p q} & =\frac{\left[N_{c}^{2}-12\right]}{2 N_{c}} d_{4}^{a b c d} .
\end{aligned}
$$

From the quadratic part of (2.8) in momentum space we find that the gluon and ghost propagators are

$$
\begin{aligned}
\left\langle A_{\mu}^{a}(p) A_{\nu}^{b}(-p)\right\rangle & =-\frac{\delta^{a b}}{\left(p^{2}\right)^{3}}\left[\eta_{\mu \nu}-(1-\alpha) \frac{p_{\mu} p_{\nu}}{p^{2}}\right], \\
\left\langle c^{a}(p) \bar{c}^{b}(-p)\right\rangle & =-\frac{\delta^{a b}}{\left(p^{2}\right)^{3}},
\end{aligned}
$$

which are formally the same as those in lower dimensions aside from the cubic power of the overall factor. This is a similar feature to other eight dimensional theories and means that the evaluation of the Feynman graphs we have to compute becomes exceedingly tedious.

While we have constructed the most general non-Abelian gauge theory based on a simple Lie group in (2.8), this is in the case where there are no masses present. The latter would not contribute to the renormalization group functions at the Wilson-Fisher fixed point which is the main reason for not considering them initially. However, one could view the presence of masses as touching the lower dimensional operators which are allowed by power counting renormalizability and which would be a staging point for connecting with the other equivalent Lagrangians for this universality class. Therefore, budgeting for nonzero masses (2.8) generalizes to

$$
\begin{aligned}
L_{m}^{(8)}= & L^{(8)}+m_{1} \bar{\psi}^{i I} \psi^{i I}-\frac{1}{4} m_{2}^{2}\left(D_{\mu} G_{\nu \sigma}^{a}\right)\left(D^{\mu} G^{a \nu \sigma}\right) \\
& -\frac{1}{2 \alpha} m_{3}^{2}\left(\partial_{\mu} \partial^{\nu} A_{\nu}^{a}\right)\left(\partial^{\mu} \partial^{\sigma} A_{\sigma}^{a}\right)-m_{3}^{2} \bar{c}^{a} \square\left(\partial^{\mu} D_{\mu} c\right)^{a} \\
& -\frac{1}{4} m_{4}^{4} G_{\mu \nu}^{a} G^{a \mu \nu}-\frac{1}{2 \alpha} m_{5}^{4}\left(\partial^{\mu} A_{\mu}^{a}\right)^{2}-m_{5}^{4} \bar{c}^{a}\left(\partial^{\mu} D_{\mu} c\right)^{a} \\
& -\frac{1}{2} m_{6}^{6} A_{\mu}^{a} A^{a \mu}+m_{6}^{6} \alpha \bar{c}^{a} c^{a}+\frac{1}{6} m_{7}^{2} f^{a b c} G_{\mu \nu}^{a} G^{b \mu \sigma} G^{c \nu} .
\end{aligned}
$$

The additional terms fall into two classes which are operators which are gauge invariant or not. In the latter case those operators are Becchi-Rouet-Stora-Tyutin (BRST) invariant. In particular it is evident that the lower dimensional operators are a reflection of the Lagrangians of the lower dimensional massless Lagrangians in the same universality class. In other words in the critical dimension of the lower dimensional Lagrangians the masses would correspond to coupling constants and hence be dimensionless in that spacetime. Implicit in (2.12) is the assumption of locality. If one ignored this and allowed for nonlocal operators then it is possible to construct a completely gauge invariant massive Lagrangian as discussed in [24]. The gluon and ghost propagators of (2.12) have Stingl forms [34], since

$$
\begin{aligned}
\left\langle A_{\mu}^{a}(p) A_{\nu}^{b}(-p)\right\rangle= & -\frac{\delta^{a b} P_{\mu \nu}(p)}{\left[\left(p^{2}\right)^{3}+m_{2}^{2}\left(p^{2}\right)^{2}+m_{4}^{4} p^{2}+m_{6}^{6}\right]} \\
& -\frac{\alpha \delta^{a b} L_{\mu \nu}(p)}{\left[\left(p^{2}\right)^{3}+m_{3}^{2}\left(p^{2}\right)^{2}+m_{5}^{2} p^{2}+\alpha m_{6}^{6}\right]}, \\
\left\langle c^{a}(p) \bar{c}^{b}(-p)\right\rangle= & -\frac{\delta^{a b}}{\left[\left(p^{2}\right)^{3}+m_{3}^{2}\left(p^{2}\right)^{2}+m_{5}^{4} p^{2}+\alpha m_{6}^{6}\right]},
\end{aligned}
$$

where 


$$
P_{\mu \nu}(p)=\eta_{\mu \nu}-\frac{p_{\mu} p_{\nu}}{p^{2}}, \quad L_{\mu \nu}(p)=\frac{p_{\mu} p_{\nu}}{p^{2}}
$$

are the respective transverse and longitudinal projection tensors. In this formulation it is apparent that the pole structure of the Faddeev-Popov ghost propagator matches that of the longitudinal part of the gluon. This ensures the cancellation of unphysical degrees of freedom within computations with the massive Lagrangian.

\section{TECHNICAL DETAILS}

The task of renormalizing (2.8) requires several technical tools some of which were applied to the determination of the two loop renormalization group functions of $L^{(6)}$. However, with the presence of gauge independent 4-point operators built from the field strength, the extraction of the $\beta$-functions of the respective coupling constants required a technique not employed in [24]. First, we note that we have constructed an automatic program to renormalize the various 2-, 3- and 4-point functions. The graphs contributing to each Green's function are generated using the FORTRAN based package QGRAF [35]. With the spinor, Lorentz and color group indices added to the electronic representation of the diagrams, each diagram is then passed to the integration routine specific to that particular $n$-point function. Once the divergences with respect to the regularization are known for each graph, the full set is summed and the renormalization constants determined automatically without the use of the subtraction method but instead using the algorithm provided in [36]. Briefly this is achieved by computing each Green's function as a function of the bare coupling constants and gauge parameter with their respective renormalized versions introduced by multiplicatively rescaling with the constant of proportionality being the renormalization constant. Specifically, at each loop order the renormalization constant associated with the Green's function is fixed by ensuring it is finite which determines the unknown counterterm at that order. Throughout this article we will consider only the $\overline{\mathrm{MS}}$ scheme and regularize the theory using dimensional regularization where the spacetime dimension $d$ is set to $d=$ $8-2 \epsilon$ and $\epsilon$ is small. It acts as the regularization parameter. To handle the significant amounts of internal algebra of this whole process, use is made of the symbolic manipulation language FORM [37,38]. It is worth noting that the renormalization of (2.8) involves 12 independent parameters as well as color and flavor parameters together with gluon and ghost propagators each of which have an exponent of 3. This means there is a significant amount of integration to be performed, compared to four dimensional QCD, for which FORM is the most efficient and practical tool for the task.

In order to construct the integration routine for each type of $n$-point function, we follow what is now a well-established procedure which is the application of the integration by parts algorithm devised by Laporta [39]. To evaluate a Feynman graph it is first written as a sum of scalar integrals where scalar products of internal and external momenta are rewritten as combinations of the inverse propagators. For cases where there is no such propagator in an integral, which is termed an irreducible, the basis of propagators is extended or completed. It transpires that for each $n$-point function at a particular loop order there is a small set of such independent completions which are called integral families. These may or may not correspond to an actual Feynman diagram topology. Irrespective of this it is the mathematical representation of the integral family which is at the center of the Laporta method. One can determine a set of general algebraic relations between integrals in each family by integration by parts and Lorentz identities. The power of the Laporta algorithm is in realizing that these relations can be solved algebraically in terms of a small set of basic or master Feynman integrals [39]. Thus if the $\epsilon$ expansion of these master integrals is known then all the Feynman integrals at that loop order can be determined. In particular this includes the specific ones which comprise each of the graphs in the $n$-point functions of interest. There are various encodings of the Laporta algorithm available but we chose to use both versions of REDUZE [40,41]. While this outlines the general approach we used, there are specific points which required attention. As we are renormalizing an eight dimensional Lagrangian we therefore need to have the master integrals in that dimension. Ordinarily the main focus in renormalization computations is four dimensions. However, we have not had to perform the explicit evaluation of master integrals by direct methods which is the normal way to determine their values. Instead we can exploit an elegant technique developed by Tarasov in $[42,43]$. By considering the graph polynomial representation of a Feynman graph, it is possible to relate a Feynman integral in $d$-dimensions in terms of a linear combination of the same integrals in $(d+2)$-dimensions. The latter, however, have several propagators with increased powers which is clearly necessary on dimensional grounds. This higher dimensional set of integrals can be reduced to a linear combination of masters in the higher dimension. One of these will be the equivalent topology as the $d$-dimensional master with the remainder of the combination being masters with a fewer number of propagators $[42,43]$. As is the case in the Laporta algorithm, some of these lower masters are integrals, such as simple bubble integrals, which are trivial to evaluate without using the Tarasov techniques. Therefore one can connect the more difficult to compute masters in $d$-dimensions with the unknown ones in $(d+2)$-dimensions. If the lower dimensional ones are available then the higher dimensional ones follow immediately. For our purposes we need to apply this connection twice since the various masters required are known in four 
dimensions. For instance, the 2-point masters to four loops have been listed in [44] while the 3-point masters for completely off-shell external legs were calculated to two loops in $[45,46]$. Also the one loop 4-point box integral is known [47]. Although we will not require the higher loop masters here, it is worth noting what has been achieved over several years.

This leads naturally to a brief discussion of the treatment of each set of $n$-point functions separately. For the 2-point functions and hence wave function renormalization constants, we carried out the renormalization to two loops. The main reason for this is that the double pole in $\epsilon$ of the two loop renormalization constant is already predetermined by the one loop computation. Therefore this provides a partial check on the leading order renormalization. For the 2-point function we used the massless Lagrangian and constructed the one and two loop masters by direct evaluation as these are straightforward bubble integrals. By contrast for the 3 -point functions, since nullifying an external leg leads to infrared issues, we had to extend the four dimensional offshell massless master 3-point function of $[44,46]$ to eight dimensions using the Tarasov method $[42,43]$. For instance, if we define the one loop triangle integral at the completely symmetric point by

$$
I(\alpha, \beta, \gamma)=\int_{k} \frac{1}{\left(k^{2}\right)^{\alpha}\left((k-p)^{2}\right)^{\beta}\left((k+q)^{2}\right)^{\gamma}}
$$

where $p$ and $q$ are the external momenta satisfying

$$
p^{2}=q^{2}=-\mu^{2}
$$

and $\int_{k}=d^{d} k /(2 \pi)^{d}$ then

$$
\begin{aligned}
\left.I(1,1,1)\right|_{d=8-2 \epsilon}= & -\mu^{2}\left[-\frac{1}{8 \epsilon}-\frac{61}{144}-\frac{2 \pi^{2}}{81}+\frac{1}{27} \psi^{\prime}\left(\frac{1}{3}\right)\right. \\
& +\left[\frac{1}{18} \psi^{\prime}\left(\frac{1}{3}\right)-\frac{895}{864}-\frac{23 \pi^{2}}{864}-\frac{2}{3} s_{3}\left(\frac{\pi}{6}\right)\right. \\
& \left.\left.+\frac{35}{5832} \pi^{3} \sqrt{3}+\frac{\pi}{216} \ln ^{2}(3) \sqrt{3}\right] \epsilon+O\left(\epsilon^{2}\right)\right]
\end{aligned}
$$

where $\psi(z)=\frac{d}{d z} \ln \Gamma(z)$ and

$$
s_{n}(z)=\frac{1}{\sqrt{3}} \mathfrak{I}\left[\operatorname{Li}_{n}\left(\frac{e^{i z}}{\sqrt{3}}\right)\right]
$$

in terms of the polylogarithm function $\mathrm{Li}_{n}(z)$. While only the simple pole in $\epsilon$ is relevant for the renormalization of (2.8), we have included the subsequent terms in the $\epsilon$ expansion for comparison with the analogous lower dimensional masters. The finite part for instance is directly correlated with the finite four dimensional master. The simple pole in (3.3) by contrast derives from the one loop bubble integrals which emerge in the Laporta reduction after the construction of the $(d+2)$-dimensional integrals from the $d$-dimensional master across two iterations. Equipped with (3.3) the three coupling constants associated with the three independent 3-point gluonic operators as well as those of the quark and ghost vertices of (2.8) were renormalized using this strategy. For the latter vertices the quark-gluon vertex renormalization, for instance, determines the renormalization constant for $g_{1}$ which can be checked in the ghost-gluon vertex computation. For the remaining two couplings in this set, $g_{2}$ and $g_{3}$, their renormalization can be determined from the gluon 3-point vertex which provides a third check on the $\beta$-function of $g_{1}$. From examining the Feynman rule for the 3 -gluon vertex it can be seen that there are three independent tensor channels to provide three independent linear relations between the renormalization constants for these couplings.

For the final part of the renormalization we have to extract the renormalization constants for the couplings associated with the purely quartic operators of each eight dimensional Lagrangian. For this we used the vacuum bubble expansion of $[48,49]$ as it was more efficient than constructing a large integration by parts database using REDUZE. This would be time consuming to construct due to the high pole propagators for the gluon and ghost. By contrast, in the vacuum bubble expansion massless propagators are recursively replaced by massive ones in such a way that the new propagators eventually produce Feynman integrals which are ultraviolet finite. Hence by Weinberg's theorem [50], these do not contribute to the overall renormalization of the Green's function and so such terms can be neglected. Subsequently the expansion terminates after a finite number of iterations. The expansion is based on the exact identity $[48,49]$,

$$
\frac{1}{(k-p)^{2}}=\frac{1}{\left[k^{2}+m^{2}\right]}+\frac{2 k p-p^{2}+m^{2}}{(k-p)^{2}\left[k^{2}+m^{2}\right]} \text {. }
$$

The contribution to the overall degree of divergence of each of the numerator pieces in the second term is less than that of the original propagator. In addition, the first term does not depend on the external momentum. So when all such terms are collected within a Feynman integral, it becomes a massive vacuum integral. Of course to produce the contributions which are purely vacuum bubbles and contain the ultraviolet divergence of the Feynman graph, the identity has to be repeated sufficient times. Once this has been achieved a simple Laporta reduction of one loop vacuum bubbles is constructed to reduce the only one loop master vacuum bubble which is a simple standard integral in eight dimensions. Another advantage of this approach is that the tensor structure arising from the external momenta together with the scalar products of external momenta derived from (3.5) emerge relatively quickly. In the summation of all the 
contributions to the gluon 4-point function such terms are central to disentangling the coupling constant renormalization constants for each of the independent quartic operators. A useful check on the procedure is the absence of the parameter of the linear covariant gauge fixing in each of the coupling constant renormalizations in the three separate color group computations we have to perform.

\section{RESULTS}

We turn now to the task of recording the results of our renormalization. First, we have followed the conventions of previous analyses [24] and note that the renormalization of the parameter of the linear covariant gauge fixing is not independent of the gluon wave function renormalization in that

$$
\gamma_{A}\left(g_{i}\right)+\gamma_{\alpha}\left(g_{i}\right)=0
$$

We have checked that this is true for all the $S U\left(N_{c}\right)$ color groups. For $S U(2)$ the anomalous dimensions of the fields are

$$
\begin{aligned}
& \left.\gamma_{A}^{S U(2)}\left(g_{i}\right)\right|_{\alpha=0}=\left[24 N_{f} g_{1}^{2}+871 g_{1}^{2}-4158 g_{1} g_{2}-1386 g_{1} g_{3}+567 g_{2}^{2}+378 g_{2} g_{3}+63 g_{3}^{2}\right] \frac{1}{1680} \\
& +\left[-57594816 N_{f} g_{1}^{4}-2754788105 g_{1}^{4}+37417536 N_{f} g_{1}^{3} g_{2}+406217016 g_{1}^{3} g_{2}+18601152 N_{f} g_{1}^{3} g_{3}\right. \\
& +191078016 g_{1}^{3} g_{3}-4398624 N_{f} g_{1}^{2} g_{2}^{2}-1747949454 g_{1}^{2} g_{2}^{2}-3900096 N_{f} g_{1}^{2} g_{2} g_{3}-2040796188 g_{1}^{2} g_{2} g_{3} \\
& -1053216 N_{f} g_{1}^{2} g_{3}^{2}-261984978 g_{1}^{2} g_{3}^{2}+137535552 g_{1}^{2} g_{4}^{2}-275071104 g_{1}^{2} g_{5}^{2}-1124500608 g_{1}^{2} g_{6}^{2} \\
& +2249001216 g_{1}^{2} g_{7}^{2}+425614392 g_{1} g_{2}^{3}+881618976 g_{1} g_{2}^{2} g_{3}+500362128 g_{1} g_{2} g_{3}^{2}+155288448 g_{1} g_{2} g_{4}^{2} \\
& +425614392 g_{1} g_{2}^{3}+881618976 g_{1} g_{2}^{2} g_{3}-310576896 g_{1} g_{2} g_{5}^{2}+234033408 g_{1} g_{2} g_{6}^{2}+425614392 g_{1} g_{2}^{3} \\
& +881618976 g_{1} g_{2}^{2} g_{3}-468066816 g_{1} g_{2} g_{7}^{2}+84640248 g_{1} g_{3}^{3}+425614392 g_{1} g_{2}^{3}+881618976 g_{1} g_{2}^{2} g_{3} \\
& +200785536 g_{1} g_{3} g_{4}^{2}-401571072 g_{1} g_{3} g_{5}^{2}+425614392 g_{1} g_{2}^{3}+881618976 g_{1} g_{2}^{2} g_{3}-21337344 g_{1} g_{3} g_{6}^{2} \\
& +42674688 g_{1} g_{3} g_{7}^{2}+425614392 g_{1} g_{2}^{3}+881618976 g_{1} g_{2}^{2} g_{3}-26643897 g_{2}^{4}-87736068 g_{2}^{3} g_{3} \\
& +425614392 g_{1} g_{2}^{3}+881618976 g_{1} g_{2}^{2} g_{3}-89488602 g_{2}^{2} g_{3}^{2}-52581312 g_{2}^{2} g_{4}^{2}+425614392 g_{1} g_{2}^{3} \\
& +881618976 g_{1} g_{2}^{2} g_{3}+105162624 g_{2}^{2} g_{5}^{2}+19813248 g_{2}^{2} g_{6}^{2}+425614392 g_{1} g_{2}^{3}+881618976 g_{1} g_{2}^{2} g_{3} \\
& -39626496 g_{2}^{2} g_{7}^{2}-35913276 g_{2} g_{3}^{3}+425614392 g_{1} g_{2}^{3}+881618976 g_{1} g_{2}^{2} g_{3}-75696768 g_{2} g_{3} g_{4}^{2} \\
& +151393536 g_{2} g_{3} g_{5}^{2}+425614392 g_{1} g_{2}^{3}+881618976 g_{1} g_{2}^{2} g_{3}+40303872 g_{2} g_{3} g_{6}^{2}-80607744 g_{2} g_{3} g_{7}^{2} \\
& +425614392 g_{1} g_{2}^{3}+881618976 g_{1} g_{2}^{2} g_{3}-5230701 g_{3}^{4}-19389888 g_{3}^{2} g_{4}^{2}+425614392 g_{1} g_{2}^{3} \\
& +881618976 g_{1} g_{2}^{2} g_{3}+38779776 g_{3}^{2} g_{5}^{2}+11233152 g_{3}^{2} g_{6}^{2}+425614392 g_{1} g_{2}^{3}+881618976 g_{1} g_{2}^{2} g_{3} \\
& \left.-22466304 g_{3}^{2} g_{7}^{2}\right] \frac{1}{338688000}+O\left(g_{i}^{6}\right) \\
& \left.\gamma_{c}^{S U(2)}\left(g_{i}\right)\right|_{\alpha=0}=-\frac{7}{24} g_{1}^{2}+\left[12312 N_{f} g_{1}^{2}-3321487 g_{1}^{2}-628614 g_{1} g_{2}-241878 g_{1} g_{3}+77301 g_{2}^{2}\right. \\
& \left.+192654 g_{2} g_{3}+108549 g_{3}^{2}\right] \frac{g_{1}^{2}}{2419200}+O\left(g_{i}^{6}\right) \\
& \left.\gamma_{\psi}^{S U(2)}\left(g_{i}\right)\right|_{\alpha=0}=\frac{7}{16} g_{1}^{2}+\left[-17352 N_{f} g_{1}^{2}+3509752 g_{1}^{2}+1722294 g_{1} g_{2}+973938 g_{1} g_{3}-196371 g_{2}^{2}\right. \\
& \left.-272034 g_{2} g_{3}-121779 g_{3}^{2}\right] \frac{g_{1}^{2}}{1612800}+O\left(g_{i}^{6}\right)
\end{aligned}
$$

in the Landau gauge which is chosen for presentational reasons. The full $\alpha$ dependent results are contained in the Supplemental Material [51]. One of the reasons for proceeding to two loops for this is as a check on the computation. The double pole in $\epsilon$ at two loops of the respective renormalization constants is not independent as it depends on the simple pole at one loop. We have verified that this is indeed the case in the explicit renormalization constants for arbitrary $\alpha$. This checks the one loop coupling constant renormalization as well as the application of the Tarasov method $[42,43]$, to raise the four and six dimension massless two loop 2-point master integrals to eight dimensions. The one loop $\beta$-functions are 


$$
\begin{aligned}
& \beta_{1}^{S U(2)}\left(g_{i}\right)=\left[24 N_{f} g_{1}^{2}-109 g_{1}^{2}-4158 g_{1} g_{2}-1386 g_{1} g_{3}+567 g_{2}^{2}+378 g_{2} g_{3}+63 g_{3}^{2}\right] \frac{g_{1}}{3360}+O\left(g_{i}^{5}\right) \\
& \beta_{2}^{S U(2)}\left(g_{i}\right)=\left[-272 N_{f} g_{1}^{3}+32152 g_{1}^{3}+216 N_{f} g_{1}^{2} g_{2}+17919 g_{1}^{2} g_{2}-19908 g_{1}^{2} g_{3}-32634 g_{1} g_{2}^{2}-2646 g_{1} g_{2} g_{3}+3528 g_{1} g_{3}^{2}\right. \\
& \left.+5103 g_{2}^{3}+2898 g_{2}^{2} g_{3}-441 g_{2} g_{3}^{2}-168 g_{3}^{3}\right] \frac{1}{10080}+O\left(g_{i}^{5}\right) \\
& \beta_{3}^{S U(2)}\left(g_{i}\right)=\left[-128 N_{f} g_{1}^{3}-18573 g_{1}^{3}+14889 g_{1}^{2} g_{2}+36 N_{f} g_{1}^{2} g_{3}+8163 g_{1}^{2} g_{3}-2520 g_{1} g_{2}^{2}-7539 g_{1} g_{2} g_{3}-777 g_{1} g_{3}^{2}\right. \\
& +5544 g_{1} g_{4}^{2}-11088 g_{1} g_{5}^{2}-3696 g_{1} g_{6}^{2}+7392 g_{1} g_{7}^{2}+819 g_{2}^{2} g_{3}+378 g_{2} g_{3}^{2}-1512 g_{2} g_{4}^{2}+3024 g_{2} g_{5}^{2} \\
& \left.+1008 g_{2} g_{6}^{2}-2016 g_{2} g_{7}^{2}-21 g_{3}^{3}-504 g_{3} g_{4}^{2}+1008 g_{3} g_{5}^{2}+336 g_{3} g_{6}^{2}-672 g_{3} g_{7}^{2}\right] \frac{1}{1680}+O\left(g_{i}^{5}\right) \\
& \beta_{4}^{S U(2)}\left(g_{i}\right)=\left[800 N_{f} g_{1}^{4}+73999 g_{1}^{4}-82068 g_{1}^{3} g_{2}-48426 g_{1}^{3} g_{3}+13734 g_{1}^{2} g_{2}^{2}+12852 g_{1}^{2} g_{2} g_{3}+3360 g_{1}^{2} g_{3}^{2}+1152 N_{f} g_{1}^{2} g_{4}^{2}\right. \\
& -89904 g_{1}^{2} g_{4}^{2}-32592 g_{1}^{2} g_{5}^{2}-113568 g_{1}^{2} g_{6}^{2}-193536 g_{1}^{2} g_{7}^{2}-42 g_{1} g_{2}^{2} g_{3}+1008 g_{1} g_{2} g_{3}^{2}-179424 g_{1} g_{2} g_{4}^{2} \\
& -15456 g_{1} g_{2} g_{5}^{2}-2688 g_{1} g_{2} g_{6}^{2}+8064 g_{1} g_{2} g_{7}^{2}+2058 g_{1} g_{3}^{3}-6720 g_{1} g_{3} g_{4}^{2}-7392 g_{1} g_{3} g_{5}^{2}-43008 g_{1} g_{3} g_{6}^{2} \\
& -45696 g_{1} g_{3} g_{7}^{2}+27216 g_{2}^{2} g_{4}^{2}+18144 g_{2} g_{3} g_{4}^{2}-903 g_{3}^{4}-23184 g_{3}^{2} g_{4}^{2}-5712 g_{3}^{2} g_{5}^{2}-12768 g_{3}^{2} g_{6}^{2}-13440 g_{3}^{2} g_{7}^{2} \\
& -169344 g_{4}^{4}-188160 g_{4}^{2} g_{5}^{2}-177408 g_{4}^{2} g_{6}^{2}-139776 g_{4}^{2} g_{7}^{2}-124992 g_{5}^{4}-145152 g_{5}^{2} g_{6}^{2}-21504 g_{5}^{2} g_{7}^{2} \\
& \left.-37632 g_{6}^{4}-43008 g_{6}^{2} g_{7}^{2}-43008 g_{7}^{4}\right] \frac{1}{40320}+O\left(g_{i}^{6}\right) \\
& \beta_{5}^{S U(2)}\left(g_{i}\right)=\left[-1192 N_{f} g_{1}^{4}-101355 g_{1}^{4}+84756 g_{1}^{3} g_{2}+19194 g_{1}^{3} g_{3}-14070 g_{1}^{2} g_{2}^{2}-16884 g_{1}^{2} g_{2} g_{3}+1848 g_{1}^{2} g_{3}^{2}+52416 g_{1}^{2} g_{4}^{2}\right. \\
& +1152 N_{f} g_{1}^{2} g_{5}^{2}+16608 g_{1}^{2} g_{5}^{2}+92064 g_{1}^{2} g_{6}^{2}+193536 g_{1}^{2} g_{7}^{2}+42 g_{1} g_{2}^{2} g_{3}+336 g_{1} g_{2} g_{3}^{2}-12096 g_{1} g_{2} g_{4}^{2} \\
& -181440 g_{1} g_{2} g_{5}^{2}+8064 g_{1} g_{2} g_{6}^{2}-8064 g_{1} g_{2} g_{7}^{2}+966 g_{1} g_{3}^{3}-11424 g_{1} g_{3} g_{4}^{2}-43008 g_{1} g_{3} g_{5}^{2}+75264 g_{1} g_{3} g_{6}^{2} \\
& +45696 g_{1} g_{3} g_{7}^{2}+27216 g_{2}^{2} g_{5}^{2}+18144 g_{2} g_{3} g_{5}^{2}-21 g_{3}^{4}+10080 g_{3}^{2} g_{4}^{2}+3360 g_{3}^{2} g_{5}^{2}+2016 g_{3}^{2} g_{6}^{2}+13440 g_{3}^{2} g_{7}^{2} \\
& -10752 g_{4}^{4}-107520 g_{4}^{2} g_{5}^{2}-37632 g_{4}^{2} g_{6}^{2}-10752 g_{4}^{2} g_{7}^{2}-12096 g_{5}^{4}-26880 g_{5}^{2} g_{6}^{2} \\
& \left.-129024 g_{5}^{2} g_{7}^{2}-26880 g_{6}^{4}-43008 g_{6}^{2} g_{7}^{2}\right] \frac{1}{40320}+O\left(g_{i}^{6}\right) \\
& \beta_{6}^{S U(2)}\left(g_{i}\right)=\left[272 N_{f} g_{1}^{4}-248207 g_{1}^{4}+14742 g_{1}^{3} g_{2}+134925 g_{1}^{3} g_{3}+231 g_{1}^{2} g_{2}^{2}-7728 g_{1}^{2} g_{2} g_{3}-1323 g_{1}^{2} g_{3}^{2}-222432 g_{1}^{2} g_{4}^{2}\right. \\
& -343392 g_{1}^{2} g_{5}^{2}+2304 N_{f} g_{1}^{2} g_{6}^{2}-1440480 g_{1}^{2} g_{6}^{2}-228480 g_{1}^{2} g_{7}^{2}+147 g_{1} g_{2}^{2} g_{3}-4326 g_{1} g_{2} g_{3}^{2}+26880 g_{1} g_{2} g_{4}^{2} \\
& +48384 g_{1} g_{2} g_{5}^{2}-204288 g_{1} g_{2} g_{6}^{2}+2688 g_{1} g_{2} g_{7}^{2}-4557 g_{1} g_{3}^{3}+52416 g_{1} g_{3} g_{4}^{2}+118272 g_{1} g_{3} g_{5}^{2} \\
& +247296 g_{1} g_{3} g_{6}^{2}+34944 g_{1} g_{3} g_{7}^{2}+54432 g_{2}^{2} g_{6}^{2}+36288 g_{2} g_{3} g_{6}^{2}-42 g_{3}^{4}+3360 g_{3}^{2} g_{4}^{2}+7392 g_{3}^{2} g_{5}^{2}+50400 g_{3}^{2} g_{6}^{2} \\
& -21504 g_{4}^{4}-80640 g_{4}^{2} g_{5}^{2}-451584 g_{4}^{2} g_{6}^{2}-21504 g_{4}^{2} g_{7}^{2}-77952 g_{5}^{4}-806400 g_{5}^{2} g_{6}^{2}-43008 g_{5}^{2} g_{7}^{2}-1666560 g_{6}^{4} \\
& \left.-301056 g_{6}^{2} g_{7}^{2}\right] \frac{1}{80640}+O\left(g_{i}^{6}\right) \\
& \beta_{7}^{S U(2)}\left(g_{i}\right)=\left[8 N_{f} g_{1}^{4}-472989 g_{1}^{4}+154266 g_{1}^{3} g_{2}+155883 g_{1}^{3} g_{3}-10647 g_{1}^{2} g_{2}^{2}-31584 g_{1}^{2} g_{2} g_{3}+651 g_{1}^{2} g_{3}^{2}+637056 g_{1}^{2} g_{4}^{2}\right. \\
& +480480 g_{1}^{2} g_{5}^{2}+1704192 g_{1}^{2} g_{6}^{2}+2304 N_{f} g_{1}^{2} g_{7}^{2}+3470496 g_{1}^{2} g_{7}^{2}-147 g_{1} g_{2}^{2} g_{3}-1050 g_{1} g_{2} g_{3}^{2}-72576 g_{1} g_{2} g_{4}^{2} \\
& -52416 g_{1} g_{2} g_{5}^{2}-202944 g_{1} g_{2} g_{6}^{2}-751296 g_{1} g_{2} g_{7}^{2}-3339 g_{1} g_{3}^{3}-124992 g_{1} g_{3} g_{4}^{2}-81984 g_{1} g_{3} g_{5}^{2} \\
& -307776 g_{1} g_{3} g_{6}^{2}-651840 g_{1} g_{3} g_{7}^{2}+54432 g_{2}^{2} g_{7}^{2}+36288 g_{2} g_{3} g_{7}^{2}-42 g_{3}^{4}-9408 g_{3}^{2} g_{4}^{2}-6048 g_{3}^{2} g_{5}^{2} \\
& -41664 g_{3}^{2} g_{6}^{2}-74592 g_{3}^{2} g_{7}^{2}-91392 g_{4}^{4}-80640 g_{4}^{2} g_{5}^{2}-408576 g_{4}^{2} g_{6}^{2}-1580544 g_{4}^{2} g_{7}^{2}-5376 g_{5}^{4}-64512 g_{5}^{2} g_{6}^{2} \\
& \left.-913920 g_{5}^{2} g_{7}^{2}-118272 g_{6}^{4}-3440640 g_{6}^{2} g_{7}^{2}-4773888 g_{7}^{4}\right] \frac{1}{80640}+O\left(g_{i}^{6}\right) \text {. }
\end{aligned}
$$

The main perturbative check on these expressions is the absence of the gauge parameter. We computed the various 4-point functions with nonzero $\alpha$ and verified that it canceled in the final Green's function as it ought since we are using the $\overline{\mathrm{MS}}$ scheme. 
The results for the case of $S U(3)$ are somewhat similar aside from the additional two couplings. We have

$$
\begin{aligned}
\left.\gamma_{A}^{S U(3)}\left(g_{i}\right)\right|_{\alpha=0}= & {\left[16 g_{1}^{2} N_{f}+871 g_{1}^{2}-4158 g_{1} g_{2}-1386 g_{1} g_{3}+567 g_{2}^{2}+378 g_{2} g_{3}+63 g_{3}^{2}\right] \frac{1}{1120} } \\
& +\left[-110877632 g_{1}^{4} N_{f}-8264364315 g_{1}^{4}+74835072 g_{1}^{3} g_{2} N_{f}+1218651048 g_{1}^{3} g_{2}+37202304 g_{1}^{3} g_{3} N_{f}\right. \\
& +573234048 g_{1}^{3} g_{3}-8797248 g_{1}^{2} g_{2}^{2} N_{f}-5243848362 g_{1}^{2} g_{2}^{2}-7800192 g_{1}^{2} g_{2} g_{3} N_{f}-6122388564 g_{1}^{2} g_{2} g_{3} \\
& -2106432 g_{1}^{2} g_{3}^{2} N_{f}-785954934 g_{1}^{2} g_{3}^{2}+275071104 g_{1}^{2} g_{4}^{2}-550142208 g_{1}^{2} g_{5}^{2}-2249001216 g_{1}^{2} g_{6}^{2} \\
& +4498002432 g_{1}^{2} g_{7}^{2}+3748335360 g_{1}^{2} g_{8}^{2}+229225920 g_{1}^{2} g_{9}^{2}+1276843176 g_{1} g_{2}^{3}+2644856928 g_{1} g_{2}^{2} g_{3} \\
& +1501086384 g_{1} g_{2} g_{3}^{2}+310576896 g_{1} g_{2} g_{4}^{2}-621153792 g_{1} g_{2} g_{5}^{2}+468066816 g_{1} g_{2} g_{6}^{2}-936133632 g_{1} g_{2} g_{7}^{2} \\
& -780111360 g_{1} g_{2} g_{8}^{2}+258814080 g_{1} g_{2} g_{9}^{2}+253920744 g_{1} g_{3}^{3}+401571072 g_{1} g_{3} g_{4}^{2}-803142144 g_{1} g_{3} g_{5}^{2} \\
& -42674688 g_{1} g_{3} g_{6}^{2}+85349376 g_{1} g_{3} g_{7}^{2}+71124480 g_{1} g_{3} g_{8}^{2}+334642560 g_{1} g_{3} g_{9}^{2}-79931691 g_{2}^{4} \\
& -263208204 g_{2}^{3} g_{3}-268465806 g_{2}^{2} g_{3}^{2}-105162624 g_{2}^{2} g_{4}^{2}+210325248 g_{2}^{2} g_{5}^{2}+39626496 g_{2}^{2} g_{6}^{2} \\
& -79252992 g_{2}^{2} g_{7}^{2}-66044160 g_{2}^{2} g_{8}^{2}-87635520 g_{2}^{2} g_{9}^{2}-107739828 g_{2} g_{3}^{3}-151393536 g_{2} g_{3} g_{4}^{2} \\
& +302787072 g_{2} g_{3} g_{5}^{2}+80607744 g_{2} g_{3} g_{6}^{2}-161215488 g_{2} g_{3} g_{7}^{2}-134346240 g_{2} g_{3} g_{8}^{2}-126161280 g_{2} g_{3} g_{9}^{2} \\
& -15692103 g_{3}^{4}-38779776 g_{3}^{2} g_{4}^{2}+77559552 g_{3}^{2} g_{5}^{2}+22466304 g_{3}^{2} g_{6}^{2}-44932608 g_{3}^{2} g_{7}^{2}-37443840 g_{3}^{2} g_{8}^{2} \\
& \left.-32316480 g_{3}^{2} g_{9}^{2}\right] \frac{1}{451584000}+O\left(g_{i}^{6}\right) \\
& -\frac{7}{16} g_{1}^{2}+\left[8208 g_{1}^{2} N_{f}-3321487 g_{1}^{2}-628614 g_{1} g_{2}-241878 g_{1} g_{3}+77301 g_{2}^{2}+192654 g_{2} g_{3}\right. \\
\left.\gamma_{c}^{S U(3)}\left(g_{i}\right)\right|_{\alpha=0}= &
\end{aligned}
$$$$
\left.+108549 g_{3}^{2}\right] \frac{g_{1}^{2}}{1075200}+O\left(g_{i}^{6}\right)
$$$$
\left.\gamma_{\psi}^{S U(3)}\left(g_{i}\right)\right|_{\alpha=0}=\frac{7}{9} g_{1}^{2}+\left[-3856 g_{1}^{2} N_{f}+1147459 g_{1}^{2}+574098 g_{1} g_{2}+324646 g_{1} g_{3}-65457 g_{2}^{2}\right.
$$$$
\left.-90678 g_{2} g_{3}-40593 g_{3}^{2}\right] \frac{g_{1}^{2}}{201600}+O\left(g_{i}^{6}\right)
$$$$
\beta_{1}^{S U(3)}\left(g_{i}\right)=\left[16 g_{1}^{2} N_{f}-109 g_{1}^{2}-4158 g_{1} g_{2}-1386 g_{1} g_{3}+567 g_{2}^{2}+378 g_{2} g_{3}+63 g_{3}^{2}\right] \frac{g_{1}}{2240}+O\left(g_{i}^{5}\right)
$$$$
\beta_{2}^{S U(3)}\left(g_{i}\right)=\left[-544 g_{1}^{3} N_{f}+96456 g_{1}^{3}+432 g_{1}^{2} g_{2} N_{f}+53757 g_{1}^{2} g_{2}-59724 g_{1}^{2} g_{3}-97902 g_{1} g_{2}^{2}-7938 g_{1} g_{2} g_{3}\right.
$$$$
\left.+10584 g_{1} g_{3}^{2}+15309 g_{2}^{3}+8694 g_{2}^{2} g_{3}-1323 g_{2} g_{3}^{2}-504 g_{3}^{3}\right] \frac{1}{20160}+O\left(g_{i}^{5}\right)
$$$$
\beta_{3}^{S U(3)}\left(g_{i}\right)=\left[-256 g_{1}^{3} N_{f}-55719 g_{1}^{3}+44667 g_{1}^{2} g_{2}+72 g_{1}^{2} g_{3} N_{f}+24489 g_{1}^{2} g_{3}-7560 g_{1} g_{2}^{2}-22617 g_{1} g_{2} g_{3}\right.
$$$$
-2331 g_{1} g_{3}^{2}+11088 g_{1} g_{4}^{2}-22176 g_{1} g_{5}^{2}-7392 g_{1} g_{6}^{2}+14784 g_{1} g_{7}^{2}+12320 g_{1} g_{8}^{2}+9240 g_{1} g_{9}^{2}+2457 g_{2}^{2} g_{3}
$$$$
+1134 g_{2} g_{3}^{2}-3024 g_{2} g_{4}^{2}+6048 g_{2} g_{5}^{2}+2016 g_{2} g_{6}^{2}-4032 g_{2} g_{7}^{2}-3360 g_{2} g_{8}^{2}-2520 g_{2} g_{9}^{2}-63 g_{3}^{3}
$$$$
\left.-1008 g_{3} g_{4}^{2}+2016 g_{3} g_{5}^{2}+672 g_{3} g_{6}^{2}-1344 g_{3} g_{7}^{2}-1120 g_{3} g_{8}^{2}-840 g_{3} g_{9}^{2}\right] \frac{1}{3360}+O\left(g_{i}^{5}\right)
$$$$
\beta_{4}^{S U(3)}\left(g_{i}\right)=\left[-784 g_{1}^{4} N_{f}-61551 g_{1}^{4}+6048 g_{1}^{3} g_{2}-65772 g_{1}^{3} g_{3}-756 g_{1}^{2} g_{2}^{2}-9072 g_{1}^{2} g_{2} g_{3}+11718 g_{1}^{2} g_{3}^{2}+3456 g_{1}^{2} g_{4}^{2} N_{f}\right.
$$$$
-168696 g_{1}^{2} g_{4}^{2}-208656 g_{1}^{2} g_{5}^{2}-417312 g_{1}^{2} g_{6}^{2}-245952 g_{1}^{2} g_{8}^{2}-85680 g_{1}^{2} g_{9}^{2}+3024 g_{1} g_{2} g_{3}^{2}-861840 g_{1} g_{2} g_{4}^{2}
$$$$
+6048 g_{1} g_{2} g_{5}^{2}+12096 g_{1} g_{2} g_{6}^{2}+6048 g_{1} g_{2} g_{9}^{2}+6804 g_{1} g_{3}^{3}-81648 g_{1} g_{3} g_{4}^{2}-30240 g_{1} g_{3} g_{5}^{2}-60480 g_{1} g_{3} g_{6}^{2}
$$$$
-88704 g_{1} g_{3} g_{8}^{2}+14112 g_{1} g_{3} g_{9}^{2}+122472 g_{2}^{2} g_{4}^{2}+81648 g_{2} g_{3} g_{4}^{2}-2079 g_{3}^{4}-58968 g_{3}^{2} g_{4}^{2}-27216 g_{3}^{2} g_{5}^{2}
$$$$
-54432 g_{3}^{2} g_{6}^{2}-20160 g_{3}^{2} g_{8}^{2}-17136 g_{3}^{2} g_{9}^{2}-870912 g_{4}^{4}-806400 g_{4}^{2} g_{5}^{2}-1016064 g_{4}^{2} g_{6}^{2}-419328 g_{4}^{2} g_{7}^{2}
$$$$
-344064 g_{4}^{2} g_{8}^{2}-365568 g_{4}^{2} g_{9}^{2}-395136 g_{5}^{4}-516096 g_{5}^{2} g_{6}^{2}-64512 g_{5}^{2} g_{7}^{2}-290304 g_{5}^{2} g_{8}^{2}-303744 g_{5}^{2} g_{9}^{2}
$$$$
-193536 g_{6}^{4}-129024 g_{6}^{2} g_{7}^{2}-150528 g_{6}^{2} g_{8}^{2}-252672 g_{6}^{2} g_{9}^{2}-129024 g_{7}^{4}-21504 g_{7}^{2} g_{9}^{2}-50176 g_{8}^{4}
$$$$
\left.-129024 g_{8}^{2} g_{9}^{2}-62720 g_{9}^{4}\right] \frac{1}{120960}+O\left(g_{i}^{6}\right)
$$ 


$$
\begin{aligned}
& \beta_{5}^{S U(3)}\left(g_{i}\right)=\left[-3576 g_{1}^{4} N_{f}-445839 g_{1}^{4}+380394 g_{1}^{3} g_{2}+97335 g_{1}^{3} g_{3}-63189 g_{1}^{2} g_{2}^{2}-74466 g_{1}^{2} g_{2} g_{3}+6363 g_{1}^{2} g_{3}^{2}\right. \\
& +157248 g_{1}^{2} g_{4}^{2}+3456 g_{1}^{2} g_{5}^{2} N_{f}+95400 g_{1}^{2} g_{5}^{2}+383040 g_{1}^{2} g_{6}^{2}+580608 g_{1}^{2} g_{7}^{2}+360864 g_{1}^{2} g_{8}^{2}+88200 g_{1}^{2} g_{9}^{2} \\
& +189 g_{1} g_{2}^{2} g_{3}+1008 g_{1} g_{2} g_{3}^{2}-36288 g_{1} g_{2} g_{4}^{2}-841680 g_{1} g_{2} g_{5}^{2}+28224 g_{1} g_{2} g_{6}^{2}-24192 g_{1} g_{2} g_{7}^{2}-20160 g_{1} g_{2} g_{8}^{2} \\
& -27216 g_{1} g_{2} g_{9}^{2}+3213 g_{1} g_{3}^{3}-34272 g_{1} g_{3} g_{4}^{2}-194544 g_{1} g_{3} g_{5}^{2}+294336 g_{1} g_{3} g_{6}^{2}+137088 g_{1} g_{3} g_{7}^{2} \\
& +69888 g_{1} g_{3} g_{8}^{2}-21504 g_{1} g_{3} g_{9}^{2}+122472 g_{2}^{2} g_{5}^{2}+81648 g_{2} g_{3} g_{5}^{2}+252 g_{3}^{4}+30240 g_{3}^{2} g_{4}^{2}+15624 g_{3}^{2} g_{5}^{2} \\
& +8064 g_{3}^{2} g_{6}^{2}+40320 g_{3}^{2} g_{7}^{2}+23520 g_{3}^{2} g_{8}^{2}+16632 g_{3}^{2} g_{9}^{2}-32256 g_{4}^{4}-322560 g_{4}^{2} g_{5}^{2}-112896 g_{4}^{2} g_{6}^{2}-32256 g_{4}^{2} g_{7}^{2} \\
& -10752 g_{4}^{2} g_{8}^{2}-21504 g_{4}^{2} g_{9}^{2}-56448 g_{5}^{4}-161280 g_{5}^{2} g_{6}^{2}-387072 g_{5}^{2} g_{7}^{2}-172032 g_{5}^{2} g_{8}^{2}-118272 g_{5}^{2} g_{9}^{2}-161280 g_{6}^{4} \\
& \left.-129024 g_{6}^{2} g_{7}^{2}-129024 g_{6}^{2} g_{8}^{2}-59136 g_{6}^{2} g_{9}^{2}-10752 g_{7}^{2} g_{9}^{2}-7168 g_{8}^{4}+25088 g_{8}^{2} g_{9}^{2}+10080 g_{9}^{4}\right] \frac{1}{120960}+O\left(g_{i}^{6}\right) \\
& \beta_{6}^{S U(3)}\left(g_{i}\right)=\left[1632 g_{1}^{4} N_{f}-1152069 g_{1}^{4}-120834 g_{1}^{3} g_{2}+778113 g_{1}^{3} g_{3}+17703 g_{1}^{2} g_{2}^{2}-10584 g_{1}^{2} g_{2} g_{3}-10899 g_{1}^{2} g_{3}^{2}\right. \\
& -1334592 g_{1}^{2} g_{4}^{2}-1846656 g_{1}^{2} g_{5}^{2}+13824 g_{1}^{2} g_{6}^{2} N_{f}-8246880 g_{1}^{2} g_{6}^{2}-1370880 g_{1}^{2} g_{7}^{2}-747264 g_{1}^{2} g_{8}^{2} \\
& -391440 g_{1}^{2} g_{9}^{2}+1323 g_{1} g_{2}^{2} g_{3}-30870 g_{1} g_{2} g_{3}^{2}+161280 g_{1} g_{2} g_{4}^{2}+298368 g_{1} g_{2} g_{5}^{2}-2407104 g_{1} g_{2} g_{6}^{2} \\
& +16128 g_{1} g_{2} g_{7}^{2}-26880 g_{1} g_{2} g_{8}^{2}+55776 g_{1} g_{2} g_{9}^{2}-29169 g_{1} g_{3}^{3}+314496 g_{1} g_{3} g_{4}^{2}+846720 g_{1} g_{3} g_{5}^{2} \\
& +1358784 g_{1} g_{3} g_{6}^{2}+209664 g_{1} g_{3} g_{7}^{2}+118272 g_{1} g_{3} g_{8}^{2}+139104 g_{1} g_{3} g_{9}^{2}+489888 g_{2}^{2} g_{6}^{2}+326592 g_{2} g_{3} g_{6}^{2} \\
& -252 g_{3}^{4}+20160 g_{3}^{2} g_{4}^{2}+48384 g_{3}^{2} g_{5}^{2}+328608 g_{3}^{2} g_{6}^{2}+7728 g_{3}^{2} g_{9}^{2}-129024 g_{4}^{4}-483840 g_{4}^{2} g_{5}^{2}-2709504 g_{4}^{2} g_{6}^{2} \\
& -129024 g_{4}^{2} g_{7}^{2}-43008 g_{4}^{2} g_{8}^{2}-86016 g_{4}^{2} g_{9}^{2}-548352 g_{5}^{4}-5160960 g_{5}^{2} g_{6}^{2}-258048 g_{5}^{2} g_{7}^{2}-258048 g_{5}^{2} g_{8}^{2} \\
& -204288 g_{5}^{2} g_{9}^{2}-10321920 g_{6}^{4}-1806336 g_{6}^{2} g_{7}^{2}-946176 g_{6}^{2} g_{8}^{2}-989184 g_{6}^{2} g_{9}^{2}-43008 g_{7}^{2} g_{9}^{2}-28672 g_{8}^{4} \\
& \left.-60928 g_{8}^{2} g_{9}^{2}-20160 g_{9}^{4}\right] \frac{1}{483840}+O\left(g_{i}^{6}\right) \\
& \beta_{7}^{S U(3)}\left(g_{i}\right)=\left[608 g_{1}^{4} N_{f}-5338695 g_{1}^{4}+1641906 g_{1}^{3} g_{2}+1839159 g_{1}^{3} g_{3}-111447 g_{1}^{2} g_{2}^{2}-343224 g_{1}^{2} g_{2} g_{3}+4851 g_{1}^{2} g_{3}^{2}\right. \\
& +8797824 g_{1}^{2} g_{4}^{2}+3673152 g_{1}^{2} g_{5}^{2}+11805696 g_{1}^{2} g_{6}^{2}+27648 g_{1}^{2} g_{7}^{2} N_{f}+59727168 g_{1}^{2} g_{7}^{2}+1537536 g_{1}^{2} g_{8}^{2} \\
& +1832880 g_{1}^{2} g_{9}^{2}-1323 g_{1} g_{2}^{2} g_{3}-17514 g_{1} g_{2} g_{3}^{2}-983808 g_{1} g_{2} g_{4}^{2}-395136 g_{1} g_{2} g_{5}^{2}-1378944 g_{1} g_{2} g_{6}^{2} \\
& -13491072 g_{1} g_{2} g_{7}^{2}-53760 g_{1} g_{2} g_{8}^{2}-213024 g_{1} g_{2} g_{9}^{2}-41895 g_{1} g_{3}^{3}-1620864 g_{1} g_{3} g_{4}^{2}-604800 g_{1} g_{3} g_{5}^{2} \\
& -2072448 g_{1} g_{3} g_{6}^{2}-11313792 g_{1} g_{3} g_{7}^{2}-231168 g_{1} g_{3} g_{8}^{2}-385056 g_{1} g_{3} g_{9}^{2}+979776 g_{2}^{2} g_{7}^{2}+653184 g_{2} g_{3} g_{7}^{2} \\
& -504 g_{3}^{4}-129024 g_{3}^{2} g_{4}^{2}-36288 g_{3}^{2} g_{5}^{2}-249984 g_{3}^{2} g_{6}^{2}-1342656 g_{3}^{2} g_{7}^{2}-25872 g_{3}^{2} g_{9}^{2}-2145024 g_{4}^{4} \\
& -1128960 g_{4}^{2} g_{5}^{2}-5225472 g_{4}^{2} g_{6}^{2}-41545728 g_{4}^{2} g_{7}^{2}-258048 g_{4}^{2} g_{8}^{2}-838656 g_{4}^{2} g_{9}^{2}-64512 g_{5}^{4}-774144 g_{5}^{2} g_{6}^{2} \\
& -14192640 g_{5}^{2} g_{7}^{2}-43008 g_{5}^{2} g_{8}^{2}-204288 g_{5}^{2} g_{9}^{2}-1419264 g_{6}^{4}-47738880 g_{6}^{2} g_{7}^{2}-516096 g_{6}^{2} g_{8}^{2}-989184 g_{6}^{2} g_{9}^{2} \\
& \left.-147603456 g_{7}^{4}-4300800 g_{7}^{2} g_{8}^{2}-8644608 g_{7}^{2} g_{9}^{2}-172032 g_{8}^{4}-60928 g_{8}^{2} g_{9}^{2}-82880 g_{9}^{4}\right] \frac{1}{967680}+O\left(g_{i}^{6}\right) \\
& \beta_{8}^{S U(3)}\left(g_{i}\right)=\left[-512 g_{1}^{4} N_{f}-2093313 g_{1}^{4}+881370 g_{1}^{3} g_{2}+530523 g_{1}^{3} g_{3}-64575 g_{1}^{2} g_{2}^{2}-166320 g_{1}^{2} g_{2} g_{3}+7875 g_{1}^{2} g_{3}^{2}\right. \\
& +1334592 g_{1}^{2} g_{4}^{2}+2487744 g_{1}^{2} g_{5}^{2}+9434880 g_{1}^{2} g_{6}^{2}+1370880 g_{1}^{2} g_{7}^{2}+9216 g_{1}^{2} g_{8}^{2} N_{f}+10474176 g_{1}^{2} g_{8}^{2} \\
& +1021440 g_{1}^{2} g_{9}^{2}-1323 g_{1} g_{2}^{2} g_{3}+6678 g_{1} g_{2} g_{3}^{2}-161280 g_{1} g_{2} g_{4}^{2}-274176 g_{1} g_{2} g_{5}^{2}-1137024 g_{1} g_{2} g_{6}^{2} \\
& -16128 g_{1} g_{2} g_{7}^{2}-3360000 g_{1} g_{2} g_{8}^{2}-110208 g_{1} g_{2} g_{9}^{2}-6363 g_{1} g_{3}^{3}-314496 g_{1} g_{3} g_{4}^{2}-435456 g_{1} g_{3} g_{5}^{2} \\
& -1733760 g_{1} g_{3} g_{6}^{2}-209664 g_{1} g_{3} g_{7}^{2}-2037504 g_{1} g_{3} g_{8}^{2}-165312 g_{1} g_{3} g_{9}^{2}+326592 g_{2}^{2} g_{8}^{2}+217728 g_{2} g_{3} g_{8}^{2} \\
& -126 g_{3}^{4}-20160 g_{3}^{2} g_{4}^{2}-36288 g_{3}^{2} g_{5}^{2}-249984 g_{3}^{2} g_{6}^{2}-197568 g_{3}^{2} g_{8}^{2}-14784 g_{3}^{2} g_{9}^{2}-1720320 g_{4}^{2} g_{8}^{2}-75264 g_{4}^{2} g_{9}^{2} \\
& -2967552 g_{5}^{2} g_{8}^{2}-129024 g_{5}^{2} g_{9}^{2}-11956224 g_{6}^{2} g_{8}^{2}-666624 g_{6}^{2} g_{9}^{2}-1204224 g_{7}^{2} g_{8}^{2}-43008 g_{7}^{2} g_{9}^{2}-9619456 g_{8}^{4} \\
& \left.-1627136 g_{8}^{2} g_{9}^{2}-47488 g_{9}^{4}\right] \frac{1}{322560}+O\left(g_{i}^{6}\right)
\end{aligned}
$$




$$
\begin{aligned}
\beta_{9}^{S U(3)}\left(g_{i}\right)= & {\left[6368 g_{1}^{4} N_{f}+830127 g_{1}^{4}-754740 g_{1}^{3} g_{2}-260442 g_{1}^{3} g_{3}+125622 g_{1}^{2} g_{2}^{2}+139860 g_{1}^{2} g_{2} g_{3}-1008 g_{1}^{2} g_{3}^{2}\right.} \\
& -314496 g_{1}^{2} g_{4}^{2}+82656 g_{1}^{2} g_{5}^{2}-124992 g_{1}^{2} g_{6}^{2}-1161216 g_{1}^{2} g_{7}^{2}-262080 g_{1}^{2} g_{8}^{2}+2304 g_{1}^{2} g_{9}^{2} N_{f}-101376 g_{1}^{2} g_{9}^{2} \\
& -378 g_{1} g_{2}^{2} g_{3}+1008 g_{1} g_{2} g_{3}^{2}+72576 g_{1} g_{2} g_{4}^{2}-100800 g_{1} g_{2} g_{5}^{2}-32256 g_{1} g_{2} g_{6}^{2}+48384 g_{1} g_{2} g_{7}^{2}+48384 g_{1} g_{2} g_{8}^{2} \\
& -536256 g_{1} g_{2} g_{9}^{2}+378 g_{1} g_{3}^{3}+68544 g_{1} g_{3} g_{4}^{2}-4032 g_{1} g_{3} g_{5}^{2}-177408 g_{1} g_{3} g_{6}^{2}-274176 g_{1} g_{3} g_{7}^{2}+86016 g_{1} g_{3} g_{8}^{2} \\
& -63840 g_{1} g_{3} g_{9}^{2}+81648 g_{2}^{2} g_{9}^{2}+54432 g_{2} g_{3} g_{9}^{2}-2583 g_{3}^{4}-60480 g_{3}^{2} g_{4}^{2}+2016 g_{3}^{2} g_{5}^{2}-4032 g_{3}^{2} g_{6}^{2}-80640 g_{3}^{2} g_{7}^{2} \\
& -22848 g_{3}^{2} g_{8}^{2}-30240 g_{3}^{2} g_{9}^{2}-107520 g_{4}^{2} g_{8}^{2}-172032 g_{4}^{2} g_{9}^{2}+451584 g_{5}^{2} g_{8}^{2}+177408 g_{5}^{2} g_{9}^{2}+43008 g_{6}^{2} g_{8}^{2} \\
& \left.+43008 g_{6}^{2} g_{9}^{2}-172032 g_{7}^{2} g_{8}^{2}-236544 g_{7}^{2} g_{9}^{2}-7168 g_{8}^{4}+28672 g_{8}^{2} g_{9}^{2}-32704 g_{9}^{4}\right] \frac{1}{80640}+O\left(g_{i}^{6}\right)
\end{aligned}
$$

for the full set or renormalization group functions.

The results for $S U\left(N_{c}\right)$ are more involved partly because of the increase in the number of independent couplings but also because of the explicit $N_{c}$ dependence. First, the Landau gauge field dimensions for $S U\left(N_{c}\right)$ are

$$
\begin{aligned}
\left.\gamma_{A}\left(g_{i}\right)\right|_{\alpha=0}= & {\left[871 N_{c} g_{1}^{2}+48 N_{f} g_{1}^{2}-4158 N_{c} g_{1} g_{2}-1386 N_{c} g_{1} g_{3}+567 N_{c} g_{2}^{2}+378 N_{c} g_{2} g_{3}+63 N_{c} g_{3}^{2}\right] \frac{1}{3360}+O\left(g_{i}^{4}\right) } \\
\left.\gamma_{c}\left(g_{i}\right)\right|_{\alpha=0}= & -\frac{7}{48} g_{1}^{2} N_{c}+\left[-3321487 N_{c} g_{1}^{2}+24624 N_{f} g_{1}^{2}-628614 N_{c} g_{1} g_{2}-241878 N_{c} g_{1} g_{3}+77301 N_{c} g_{2}^{2}\right. \\
& \left.+192654 N_{c} g_{2} g_{3}+108549 N_{c} g_{3}^{2}\right] \frac{g_{1}^{2} N_{c}}{9676800}+O\left(g_{i}^{6}\right) \\
\left.\gamma_{\psi}\left(g_{i}\right)\right|_{\alpha=0}= & \frac{7\left[N_{c}^{2}-1\right]}{24 N_{c}} g_{1}^{2}+\left[3388477 N_{c}^{4} g_{1}^{2}-34704 N_{c}^{3} N_{f} g_{1}^{2}-2903377 N_{c}^{2} g_{1}^{2}+34704 N_{c} N_{f} g_{1}^{2}-485100 g_{1}^{2}\right. \\
& +1722294 N_{c}^{4} g_{1} g_{2}-1722294 N_{c}^{2} g_{1} g_{2}+973938 N_{c}^{4} g_{1} g_{3}-973938 N_{c}^{2} g_{1} g_{3}-196371 N_{c}^{4} g_{2}^{2}+196371 N_{c}^{2} g_{2}^{2} \\
& \left.-272034 N_{c}^{4} g_{2} g_{3}+272034 N_{c}^{2} g_{2} g_{3}-121779 N_{c}^{4} g_{3}^{2}+121779 N_{c}^{2} g_{3}^{2}\right] \frac{g_{1}^{2}}{4838400 N_{c}^{2}}+O\left(g_{i}^{6}\right)
\end{aligned}
$$

where we only present the two loop terms of the ghost and quark for compactness. That for $\gamma_{A}\left(g_{i}\right)$ is given in the Supplemental Material together with all the other renormalization group functions. For the $\beta$-functions we found

$$
\begin{aligned}
& \beta_{1}\left(g_{i}\right)=\frac{3}{320} N_{c} g_{1} g_{3}^{2}+\frac{9}{160} N_{c} g_{1} g_{2} g_{3}+\frac{27}{320} N_{c} g_{1} g_{2}^{2}-\frac{33}{160} N_{c} g_{1}^{2} g_{3}-\frac{99}{160} N_{c} g_{1}^{2} g_{2}-\frac{109}{6720} N_{c} g_{1}^{3}+\frac{1}{140} N_{f} g_{1}^{3}+O\left(g_{i}^{5}\right) \\
& \beta_{2}\left(g_{i}\right)=-\frac{1}{120} N_{c} g_{3}^{3}-\frac{7}{320} N_{c} g_{2} g_{3}^{2}+\frac{23}{160} N_{c} g_{2}^{2} g_{3}+\frac{81}{320} N_{c} g_{2}^{3}+\frac{7}{40} N_{c} g_{1} g_{3}^{2}-\frac{21}{160} N_{c} g_{1} g_{2} g_{3}-\frac{259}{160} N_{c} g_{1} g_{2}^{2}-\frac{79}{80} N_{c} g_{1}^{2} g_{3} \\
& +\frac{1991}{2240} N_{c} g_{1}^{2} g_{2}+\frac{4019}{2520} N_{c} g_{1}^{3}+\frac{3}{140} N_{f} g_{1}^{2} g_{2}-\frac{17}{630} N_{f} g_{1}^{3}+O\left(g_{i}^{5}\right) \\
& \beta_{3}\left(g_{i}\right)=-\frac{6}{5 N_{c}} g_{3} g_{11}^{2}-\frac{2}{5 N_{c}} g_{3} g_{10}^{2}+\frac{3}{5 N_{c}} g_{3} g_{9}^{2}+\frac{4}{5 N_{c}} g_{3} g_{8}^{2}-\frac{18}{5 N_{c}} g_{2} g_{11}^{2}-\frac{6}{5 N_{c}} g_{2} g_{10}^{2}+\frac{9}{5 N_{c}} g_{2} g_{9}^{2}+\frac{12}{5 N_{c}} g_{2} g_{8}^{2}+\frac{66}{5 N_{c}} g_{1} g_{11}^{2} \\
& +\frac{22}{5 N_{c}} g_{1} g_{10}^{2}-\frac{33}{5 N_{c}} g_{1} g_{9}^{2}-\frac{44}{5 N_{c}} g_{1} g_{8}^{2}-\frac{2}{5} g_{3} g_{7}^{2}+\frac{1}{5} g_{3} g_{6}^{2}+\frac{3}{5} g_{3} g_{5}^{2}-\frac{3}{10} g_{3} g_{4}^{2}-\frac{6}{5} g_{2} g_{7}^{2}+\frac{3}{5} g_{2} g_{6}^{2}+\frac{9}{5} g_{2} g_{5}^{2}-\frac{9}{10} g_{2} g_{4}^{2} \\
& +\frac{22}{5} g_{1} g_{7}^{2}-\frac{11}{5} g_{1} g_{6}^{2}-\frac{33}{5} g_{1} g_{5}^{2}+\frac{33}{10} g_{1} g_{4}^{2}+\frac{3}{10} N_{c} g_{3} g_{11}^{2}+\frac{1}{10} N_{c} g_{3} g_{10}^{2}-\frac{3}{20} N_{c} g_{3} g_{9}^{2}-\frac{1}{5} N_{c} g_{3} g_{8}^{2}-\frac{1}{160} N_{c} g_{3}^{3} \\
& +\frac{9}{10} N_{c} g_{2} g_{11}^{2}+\frac{3}{10} N_{c} g_{2} g_{10}^{2}-\frac{9}{20} N_{c} g_{2} g_{9}^{2}-\frac{3}{5} N_{c} g_{2} g_{8}^{2}+\frac{9}{80} N_{c} g_{2} g_{3}^{2}+\frac{39}{160} N_{c} g_{2}^{2} g_{3}-\frac{33}{10} N_{c} g_{1} g_{11}^{2}-\frac{11}{10} N_{c} g_{1} g_{10}^{2} \\
& +\frac{33}{20} N_{c} g_{1} g_{9}^{2}+\frac{11}{5} N_{c} g_{1} g_{8}^{2}-\frac{37}{160} N_{c} g_{1} g_{3}^{2}-\frac{359}{160} N_{c} g_{1} g_{2} g_{3}-\frac{3}{4} N_{c} g_{1} g_{2}^{2}+\frac{2721}{1120} N_{c} g_{1}^{2} g_{3}+\frac{709}{160} N_{c} g_{1}^{2} g_{2}-\frac{6191}{1120} N_{c} g_{1}^{3} \\
& +\frac{3}{140} N_{f} g_{1}^{2} g_{3}-\frac{8}{105} N_{f} g_{1}^{3}+O\left(g_{i}^{5}\right)
\end{aligned}
$$


$\beta_{4}\left(g_{i}\right)=\frac{92}{5 N_{c}^{2}} g_{11}^{4}+\frac{184}{15 N_{c}^{2}} g_{10}^{2} g_{11}^{2}+\frac{8}{5 N_{c}^{2}} g_{10}^{4}+\frac{76}{5 N_{c}^{2}} g_{9}^{2} g_{11}^{2}+\frac{24}{5 N_{c}^{2}} g_{9}^{2} g_{10}^{2}+\frac{22}{3 N_{c}^{2}} g_{9}^{4}+\frac{208}{15 N_{c}^{2}} g_{8}^{2} g_{11}^{2}+\frac{64}{15 N_{c}^{2}} g_{8}^{2} g_{10}^{2}+\frac{224}{15 N_{c}^{2}} g_{8}^{2} g_{9}^{2}$

$+\frac{32}{5 N_{c}^{2}} g_{8}^{4}-\frac{1}{30 N_{c}^{2}} g_{3}^{2} g_{11}^{2}+\frac{1}{15 N_{c}^{2}} g_{3}^{2} g_{10}^{2}+\frac{1}{N_{c}^{2}} g_{3}^{2} g_{9}^{2}+\frac{4}{3 N_{c}^{2}} g_{3}^{2} g_{8}^{2}+\frac{1}{15 N_{c}^{2}} g_{1} g_{3} g_{11}^{2}+\frac{44}{15 N_{c}^{2}} g_{1} g_{3} g_{10}^{2}-\frac{17}{15 N_{c}^{2}} g_{1} g_{3} g_{9}^{2}$

$+\frac{68}{15 N_{c}^{2}} g_{1} g_{3} g_{8}^{2}+\frac{5}{3 N_{c}^{2}} g_{1} g_{2} g_{11}^{2}+\frac{8}{15 N_{c}^{2}} g_{1} g_{2} g_{10}^{2}-\frac{6}{5 N_{c}^{2}} g_{1} g_{2} g_{9}^{2}-\frac{4}{5 N_{c}^{2}} g_{1} g_{2} g_{8}^{2}-\frac{41}{30 N_{c}^{2}} g_{1}^{2} g_{11}^{2}+\frac{31}{15 N_{c}^{2}} g_{1}^{2} g_{10}^{2}$

$+\frac{26}{5 N_{c}^{2}} g_{1}^{2} g_{9}^{2}+\frac{96}{5 N_{c}^{2}} g_{1}^{2} g_{8}^{2}+\frac{32}{5 N_{c}} g_{6}^{2} g_{11}^{2}+\frac{16}{15 N_{c}} g_{6}^{2} g_{10}^{2}+\frac{56}{15 N_{c}} g_{6}^{2} g_{9}^{2}+\frac{32}{15 N_{c}} g_{6}^{2} g_{8}^{2}+\frac{16}{5 N_{c}} g_{5}^{2} g_{11}^{2}+\frac{8}{15 N_{c}} g_{5}^{2} g_{10}^{2}$

$+\frac{28}{15 N_{c}} g_{5}^{2} g_{9}^{2}+\frac{16}{15 N_{c}} g_{5}^{2} g_{8}^{2}+\frac{48}{5 N_{c}} g_{4}^{2} g_{11}^{2}+\frac{16}{5 N_{c}} g_{4}^{2} g_{10}^{2}+\frac{32}{5 N_{c}} g_{4}^{2} g_{9}^{2}+\frac{32}{5 N_{c}} g_{4}^{2} g_{8}^{2}-\frac{2}{3 N_{c}} g_{3}^{2} g_{7}^{2}-\frac{1}{30 N_{c}} g_{3}^{2} g_{6}^{2}$

$+\frac{1}{60 N_{c}} g_{3}^{2} g_{5}^{2}-\frac{1}{2 N_{c}} g_{3}^{2} g_{4}^{2}-\frac{34}{15 N_{c}} g_{1} g_{3} g_{7}^{2}-\frac{22}{15 N_{c}} g_{1} g_{3} g_{6}^{2}-\frac{1}{30 N_{c}} g_{1} g_{3} g_{5}^{2}+\frac{17}{30 N_{c}} g_{1} g_{3} g_{4}^{2}+\frac{2}{5 N_{c}} g_{1} g_{2} g_{7}^{2}$

$-\frac{4}{15 N_{c}} g_{1} g_{2} g_{6}^{2}-\frac{5}{6 N_{c}} g_{1} g_{2} g_{5}^{2}+\frac{3}{5 N_{c}} g_{1} g_{2} g_{4}^{2}-\frac{48}{5 N_{c}} g_{1}^{2} g_{7}^{2}-\frac{31}{30 N_{c}} g_{1}^{2} g_{6}^{2}+\frac{41}{60 N_{c}} g_{1}^{2} g_{5}^{2}-\frac{13}{5 N_{c}} g_{1}^{2} g_{4}^{2}-\frac{23}{5} g_{11}^{4}-\frac{46}{15} g_{10}^{2} g_{11}^{2}$

$-\frac{2}{5} g_{10}^{4}-\frac{19}{5} g_{9}^{2} g_{11}^{2}-\frac{6}{5} g_{9}^{2} g_{10}^{2}-\frac{11}{6} g_{9}^{4}-\frac{52}{15} g_{8}^{2} g_{11}^{2}-\frac{16}{15} g_{8}^{2} g_{10}^{2}-\frac{56}{15} g_{8}^{2} g_{9}^{2}-\frac{8}{5} g_{8}^{4}-\frac{16}{15} g_{7}^{4}-\frac{16}{15} g_{6}^{2} g_{7}^{2}-\frac{2}{5} g_{6}^{4}-\frac{8}{15} g_{5}^{2} g_{7}^{2}$

$-\frac{46}{15} g_{5}^{2} g_{6}^{2}-\frac{89}{30} g_{5}^{4}-\frac{52}{15} g_{4}^{2} g_{7}^{2}-\frac{6}{5} g_{4}^{2} g_{6}^{2}-\frac{46}{15} g_{4}^{2} g_{5}^{2}-\frac{9}{5} g_{4}^{4}+\frac{1}{120} g_{3}^{2} g_{11}^{2}-\frac{1}{60} g_{3}^{2} g_{10}^{2}-\frac{1}{4} g_{3}^{2} g_{9}^{2}-\frac{1}{3} g_{3}^{2} g_{8}^{2}-\frac{43}{1920} g_{3}^{4}$

$-\frac{1}{60} g_{1} g_{3} g_{11}^{2}-\frac{11}{15} g_{1} g_{3} g_{10}^{2}+\frac{17}{60} g_{1} g_{3} g_{9}^{2}-\frac{17}{15} g_{1} g_{3} g_{8}^{2}+\frac{49}{960} g_{1} g_{3}^{3}-\frac{5}{12} g_{1} g_{2} g_{11}^{2}-\frac{2}{15} g_{1} g_{2} g_{10}^{2}+\frac{3}{10} g_{1} g_{2} g_{9}^{2}+\frac{1}{5} g_{1} g_{2} g_{8}^{2}$

$+\frac{1}{40} g_{1} g_{2} g_{3}^{2}-\frac{1}{960} g_{1} g_{2}^{2} g_{3}+\frac{41}{120} g_{1}^{2} g_{11}^{2}-\frac{31}{60} g_{1}^{2} g_{10}^{2}-\frac{13}{10} g_{1}^{2} g_{9}^{2}-\frac{24}{5} g_{1}^{2} g_{8}^{2}+\frac{1}{12} g_{1}^{2} g_{3}^{2}+\frac{51}{160} g_{1}^{2} g_{2} g_{3}+\frac{109}{320} g_{1}^{2} g_{2}^{2}$

$-\frac{1153}{960} g_{1}^{3} g_{3}-\frac{977}{480} g_{1}^{3} g_{2}+\frac{73999}{40320} g_{1}^{4}-\frac{8}{5} N_{c} g_{6}^{2} g_{11}^{2}-\frac{4}{15} N_{c} g_{6}^{2} g_{10}^{2}-\frac{14}{15} N_{c} g_{6}^{2} g_{9}^{2}-\frac{8}{15} N_{c} g_{6}^{2} g_{8}^{2}-\frac{4}{5} N_{c} g_{5}^{2} g_{11}^{2}$

$-\frac{2}{15} N_{c} g_{5}^{2} g_{10}^{2}-\frac{7}{15} N_{c} g_{5}^{2} g_{9}^{2}-\frac{4}{15} N_{c} g_{5}^{2} g_{8}^{2}-\frac{12}{5} N_{c} g_{4}^{2} g_{11}^{2}-\frac{4}{5} N_{c} g_{4}^{2} g_{10}^{2}-\frac{8}{5} N_{c} g_{4}^{2} g_{9}^{2}-\frac{8}{5} N_{c} g_{4}^{2} g_{8}^{2}-\frac{3}{20} N_{c} g_{3}^{2} g_{6}^{2}$

$-\frac{3}{40} N_{c} g_{3}^{2} g_{5}^{2}-\frac{13}{80} N_{c} g_{3}^{2} g_{4}^{2}+\frac{9}{40} N_{c} g_{2} g_{3} g_{4}^{2}+\frac{27}{80} N_{c} g_{2}^{2} g_{4}^{2}-\frac{1}{6} N_{c} g_{1} g_{3} g_{6}^{2}-\frac{1}{12} N_{c} g_{1} g_{3} g_{5}^{2}-\frac{9}{40} N_{c} g_{1} g_{3} g_{4}^{2}$

$+\frac{1}{30} N_{c} g_{1} g_{2} g_{6}^{2}+\frac{1}{60} N_{c} g_{1} g_{2} g_{5}^{2}-\frac{19}{8} N_{c} g_{1} g_{2} g_{4}^{2}-\frac{23}{20} N_{c} g_{1}^{2} g_{6}^{2}-\frac{23}{40} N_{c} g_{1}^{2} g_{5}^{2}-\frac{781}{1680} N_{c} g_{1}^{2} g_{4}^{2}-\frac{2}{15} N_{c}^{2} g_{6}^{4}-\frac{2}{15} N_{c}^{2} g_{5}^{2} g_{6}^{2}$

$-\frac{1}{30} N_{c}^{2} g_{5}^{4}-\frac{4}{5} N_{c}^{2} g_{4}^{2} g_{6}^{2}-\frac{2}{5} N_{c}^{2} g_{4}^{2} g_{5}^{2}-\frac{3}{5} N_{c}^{2} g_{4}^{4}+\frac{5}{126 N_{c}} N_{f} g_{1}^{4}+\frac{1}{35} N_{f} g_{1}^{2} g_{4}^{2}+O\left(g_{i}^{6}\right)$

$\beta_{5}\left(g_{i}\right)=\frac{4}{5 N_{c}^{2}} g_{11}^{4}+\frac{8}{15 N_{c}^{2}} g_{10}^{2} g_{11}^{2}+\frac{8}{15 N_{c}^{2}} g_{10}^{4}+\frac{52}{5 N_{c}^{2}} g_{9}^{2} g_{11}^{2}+\frac{56}{15 N_{c}^{2}} g_{9}^{2} g_{10}^{2}+\frac{6}{5 N_{c}^{2}} g_{9}^{4}+\frac{176}{15 N_{c}^{2}} g_{8}^{2} g_{11}^{2}+\frac{64}{15 N_{c}^{2}} g_{8}^{2} g_{10}^{2}$

$+\frac{32}{15 N_{c}^{2}} g_{8}^{2} g_{9}^{2}+\frac{32}{15 N_{c}^{2}} g_{8}^{4}+\frac{1}{30 N_{c}^{2}} g_{3}^{2} g_{11}^{2}-\frac{1}{15 N_{c}^{2}} g_{3}^{2} g_{10}^{2}-\frac{1}{N_{c}^{2}} g_{3}^{2} g_{9}^{2}-\frac{4}{3 N_{c}^{2}} g_{3}^{2} g_{8}^{2}-\frac{1}{15 N_{c}^{2}} g_{1} g_{3} g_{11}^{2}-\frac{44}{15 N_{c}^{2}} g_{1} g_{3} g_{10}^{2}$

$+\frac{17}{15 N_{c}^{2}} g_{1} g_{3} g_{9}^{2}-\frac{68}{15 N_{c}^{2}} g_{1} g_{3} g_{8}^{2}-\frac{5}{3 N_{c}^{2}} g_{1} g_{2} g_{11}^{2}-\frac{8}{15 N_{c}^{2}} g_{1} g_{2} g_{10}^{2}+\frac{6}{5 N_{c}^{2}} g_{1} g_{2} g_{9}^{2}+\frac{4}{5 N_{c}^{2}} g_{1} g_{2} g_{8}^{2}+\frac{41}{30 N_{c}^{2}} g_{1}^{2} g_{11}^{2}$

$-\frac{31}{15 N_{c}^{2}} g_{1}^{2} g_{10}^{2}-\frac{26}{5 N_{c}^{2}} g_{1}^{2} g_{9}^{2}-\frac{96}{5 N_{c}^{2}} g_{1}^{2} g_{8}^{2}+\frac{16}{15 N_{c}} g_{6}^{2} g_{10}^{2}+\frac{8}{15 N_{c}} g_{6}^{2} g_{9}^{2}+\frac{32}{15 N_{c}} g_{6}^{2} g_{8}^{2}+\frac{8}{15 N_{c}} g_{5}^{2} g_{10}^{2}+\frac{4}{15 N_{c}} g_{5}^{2} g_{9}^{2}$

$+\frac{16}{15 N_{c}} g_{5}^{2} g_{8}^{2}+\frac{2}{3 N_{c}} g_{3}^{2} g_{7}^{2}+\frac{1}{30 N_{c}} g_{3}^{2} g_{6}^{2}-\frac{1}{60 N_{c}} g_{3}^{2} g_{5}^{2}+\frac{1}{2 N_{c}} g_{3}^{2} g_{4}^{2}+\frac{34}{15 N_{c}} g_{1} g_{3} g_{7}^{2}+\frac{22}{15 N_{c}} g_{1} g_{3} g_{6}^{2}+\frac{1}{30 N_{c}} g_{1} g_{3} g_{5}^{2}$

$-\frac{17}{30 N_{c}} g_{1} g_{3} g_{4}^{2}-\frac{2}{5 N_{c}} g_{1} g_{2} g_{7}^{2}+\frac{4}{15 N_{c}} g_{1} g_{2} g_{6}^{2}+\frac{5}{6 N_{c}} g_{1} g_{2} g_{5}^{2}-\frac{3}{5 N_{c}} g_{1} g_{2} g_{4}^{2}+\frac{48}{5 N_{c}} g_{1}^{2} g_{7}^{2}+\frac{31}{30 N_{c}} g_{1}^{2} g_{6}^{2}-\frac{41}{60 N_{c}} g_{1}^{2} g_{5}^{2}$

$+\frac{13}{5 N_{c}} g_{1}^{2} g_{4}^{2}-\frac{1}{5} g_{11}^{4}-\frac{2}{15} g_{10}^{2} g_{11}^{2}-\frac{2}{15} g_{10}^{4}-\frac{13}{5} g_{9}^{2} g_{11}^{2}-\frac{14}{15} g_{9}^{2} g_{10}^{2}-\frac{3}{10} g_{9}^{4}-\frac{44}{15} g_{8}^{2} g_{11}^{2}-\frac{16}{15} g_{8}^{2} g_{10}^{2}-\frac{8}{15} g_{8}^{2} g_{9}^{2}-\frac{8}{15} g_{8}^{4}$ 
$-\frac{16}{15} g_{6}^{2} g_{7}^{2}-\frac{2}{15} g_{6}^{4}-\frac{16}{5} g_{5}^{2} g_{7}^{2}-\frac{2}{15} g_{5}^{2} g_{6}^{2}-\frac{1}{6} g_{5}^{4}-\frac{4}{15} g_{4}^{2} g_{7}^{2}-\frac{14}{15} g_{4}^{2} g_{6}^{2}-\frac{8}{3} g_{4}^{2} g_{5}^{2}-\frac{4}{15} g_{4}^{4}-\frac{1}{120} g_{3}^{2} g_{11}^{2}+\frac{1}{60} g_{3}^{2} g_{10}^{2}$ $+\frac{1}{4} g_{3}^{2} g_{9}^{2}+\frac{1}{3} g_{3}^{2} g_{8}^{2}-\frac{1}{1920} g_{3}^{4}+\frac{1}{60} g_{1} g_{3} g_{11}^{2}+\frac{11}{15} g_{1} g_{3} g_{10}^{2}-\frac{17}{60} g_{1} g_{3} g_{9}^{2}+\frac{17}{15} g_{1} g_{3} g_{8}^{2}+\frac{23}{960} g_{1} g_{3}^{3}+\frac{5}{12} g_{1} g_{2} g_{11}^{2}$ $+\frac{2}{15} g_{1} g_{2} g_{10}^{2}-\frac{3}{10} g_{1} g_{2} g_{9}^{2}-\frac{1}{5} g_{1} g_{2} g_{8}^{2}+\frac{1}{120} g_{1} g_{2} g_{3}^{2}+\frac{1}{960} g_{1} g_{2}^{2} g_{3}-\frac{41}{120} g_{1}^{2} g_{11}^{2}+\frac{31}{60} g_{1}^{2} g_{10}^{2}+\frac{13}{10} g_{1}^{2} g_{9}^{2}+\frac{24}{5} g_{1}^{2} g_{8}^{2}$ $+\frac{11}{240} g_{1}^{2} g_{3}^{2}-\frac{67}{160} g_{1}^{2} g_{2} g_{3}-\frac{67}{192} g_{1}^{2} g_{2}^{2}+\frac{457}{960} g_{1}^{3} g_{3}+\frac{1009}{480} g_{1}^{3} g_{2}-\frac{6757}{2688} g_{1}^{4}-\frac{4}{15} N_{c} g_{6}^{2} g_{10}^{2}-\frac{2}{15} N_{c} g_{6}^{2} g_{9}^{2}-\frac{8}{15} N_{c} g_{6}^{2} g_{8}^{2}$ $-\frac{2}{15} N_{c} g_{5}^{2} g_{10}^{2}-\frac{1}{15} N_{c} g_{5}^{2} g_{9}^{2}-\frac{4}{15} N_{c} g_{5}^{2} g_{8}^{2}+\frac{1}{60} N_{c} g_{3}^{2} g_{6}^{2}+\frac{11}{240} N_{c} g_{3}^{2} g_{5}^{2}+\frac{9}{40} N_{c} g_{2} g_{3} g_{5}^{2}+\frac{27}{80} N_{c} g_{2}^{2} g_{5}^{2}+\frac{17}{30} N_{c} g_{1} g_{3} g_{6}^{2}$ $-\frac{13}{24} N_{c} g_{1} g_{3} g_{5}^{2}+\frac{1}{30} N_{c} g_{1} g_{2} g_{6}^{2}-\frac{59}{24} N_{c} g_{1} g_{2} g_{5}^{2}+\frac{53}{60} N_{c} g_{1}^{2} g_{6}^{2}+\frac{211}{560} N_{c} g_{1}^{2} g_{5}^{2}-\frac{2}{15} N_{c}^{2} g_{6}^{4}-\frac{2}{15} N_{c}^{2} g_{5}^{2} g_{6}^{2}-\frac{1}{30} N_{c}^{2} g_{5}^{4}$ $-\frac{149}{2520 N_{c}} N_{f} g_{1}^{4}+\frac{1}{35} N_{f} g_{1}^{2} g_{5}^{2}+O\left(g_{i}^{6}\right)$

$\beta_{6}\left(g_{i}\right)=\frac{52}{15 N_{c}^{2}} g_{11}^{4}+\frac{568}{15 N_{c}^{2}} g_{10}^{2} g_{11}^{2}+\frac{1208}{15 N_{c}^{2}} g_{10}^{4}+\frac{56}{15 N_{c}^{2}} g_{9}^{2} g_{11}^{2}+\frac{112}{5 N_{c}^{2}} g_{9}^{2} g_{10}^{2}+\frac{6}{5 N_{c}^{2}} g_{9}^{4}+\frac{16}{15 N_{c}^{2}} g_{8}^{2} g_{11}^{2}+\frac{224}{15 N_{c}^{2}} g_{8}^{2} g_{10}^{2}$ $+\frac{32}{15 N_{c}^{2}} g_{8}^{2} g_{9}^{2}+\frac{32}{15 N_{c}^{2}} g_{8}^{4}-\frac{3}{10 N_{c}^{2}} g_{3}^{2} g_{11}^{2}-\frac{31}{15 N_{c}^{2}} g_{3}^{2} g_{10}^{2}-\frac{1}{6 N_{c}^{2}} g_{3}^{2} g_{9}^{2}-\frac{18}{5 N_{c}^{2}} g_{1} g_{3} g_{11}^{2}-\frac{43}{3 N_{c}^{2}} g_{1} g_{3} g_{10}^{2}-\frac{13}{5 N_{c}^{2}} g_{1} g_{3} g_{9}^{2}$ $-\frac{26}{15 N_{c}^{2}} g_{1} g_{3} g_{8}^{2}-\frac{34}{15 N_{c}^{2}} g_{1} g_{2} g_{11}^{2}-\frac{47}{5 N_{c}^{2}} g_{1} g_{2} g_{10}^{2}-\frac{4}{3 N_{c}^{2}} g_{1} g_{2} g_{9}^{2}-\frac{2}{15 N_{c}^{2}} g_{1} g_{2} g_{8}^{2}+\frac{617}{30 N_{c}^{2}} g_{1}^{2} g_{11}^{2}+\frac{78}{N_{c}^{2}} g_{1}^{2} g_{10}^{2}$ $+\frac{331}{30 N_{c}^{2}} g_{1}^{2} g_{9}^{2}+\frac{34}{3 N_{c}^{2}} g_{1}^{2} g_{8}^{2}+\frac{16}{15 N_{c}} g_{6}^{2} g_{10}^{2}+\frac{8}{15 N_{c}} g_{6}^{2} g_{9}^{2}+\frac{32}{15 N_{c}} g_{6}^{2} g_{8}^{2}+\frac{8}{15 N_{c}} g_{5}^{2} g_{10}^{2}+\frac{4}{15 N_{c}} g_{5}^{2} g_{9}^{2}+\frac{16}{15 N_{c}} g_{5}^{2} g_{8}^{2}$ $+\frac{31}{30 N_{c}} g_{3}^{2} g_{6}^{2}+\frac{3}{20 N_{c}} g_{3}^{2} g_{5}^{2}+\frac{1}{12 N_{c}} g_{3}^{2} g_{4}^{2}+\frac{13}{15 N_{c}} g_{1} g_{3} g_{7}^{2}+\frac{43}{6 N_{c}} g_{1} g_{3} g_{6}^{2}+\frac{9}{5 N_{c}} g_{1} g_{3} g_{5}^{2}$ $+\frac{13}{10 N_{c}} g_{1} g_{3} g_{4}^{2}+\frac{1}{15 N_{c}} g_{1} g_{2} g_{7}^{2}+\frac{47}{10 N_{c}} g_{1} g_{2} g_{6}^{2}+\frac{17}{15 N_{c}} g_{1} g_{2} g_{5}^{2}+\frac{2}{3 N_{c}} g_{1} g_{2} g_{4}^{2}-\frac{17}{3 N_{c}} g_{1}^{2} g_{7}^{2}-\frac{39 N_{c} 2}{g} g_{6}^{2}-\frac{617}{60 N_{c}} g_{1}^{2} g_{5}^{2}$ $-\frac{331}{60 N_{c}} g_{1}^{2} g_{4}^{2}-\frac{13}{15} g_{11}^{4}-\frac{142}{15} g_{10}^{2} g_{11}^{2}-\frac{302}{15} g_{10}^{4}-\frac{14}{15} g_{9}^{2} g_{11}^{2}-\frac{28}{5} g_{9}^{2} g_{10}^{2}-\frac{3}{10} g_{9}^{4}-\frac{4}{15} g_{8}^{2} g_{11}^{2}-\frac{56}{15} g_{8}^{2} g_{10}^{2}-\frac{8}{15} g_{8}^{2} g_{9}^{2}$ $-\frac{8}{15} g_{8}^{4}-\frac{56}{15} g_{6}^{2} g_{7}^{2}-\frac{302}{15} g_{6}^{4}-\frac{8}{15} g_{5}^{2} g_{7}^{2}-\frac{142}{15} g_{5}^{2} g_{6}^{2}-\frac{5}{6} g_{5}^{4}-\frac{4}{15} g_{4}^{2} g_{7}^{2}-\frac{28}{5} g_{4}^{2} g_{6}^{2}-g_{4}^{2} g_{5}^{2}-\frac{4}{15} g_{4}^{4}+\frac{3}{40} g_{3}^{2} g_{11}^{2}$ $+\frac{31}{60} g_{3}^{2} g_{10}^{2}+\frac{1}{24} g_{3}^{2} g_{9}^{2}-\frac{1}{1920} g_{3}^{4}+\frac{9}{10} g_{1} g_{3} g_{11}^{2}+\frac{43}{12} g_{1} g_{3} g_{10}^{2}+\frac{13}{20} g_{1} g_{3} g_{9}^{2}+\frac{13}{30} g_{1} g_{3} g_{8}^{2}-\frac{217}{3840} g_{1} g_{3}^{3}+\frac{17}{30} g_{1} g_{2} g_{11}^{2}$ $+\frac{47}{20} g_{1} g_{2} g_{10}^{2}+\frac{1}{3} g_{1} g_{2} g_{9}^{2}+\frac{1}{30} g_{1} g_{2} g_{8}^{2}-\frac{103}{1920} g_{1} g_{2} g_{3}^{2}+\frac{7}{3840} g_{1} g_{2}^{2} g_{3}-\frac{617}{120} g_{1}^{2} g_{11}^{2}-\frac{39}{2} g_{1}^{2} g_{10}^{2}-\frac{331}{120} g_{1}^{2} g_{9}^{2}-\frac{17}{6} g_{1}^{2} g_{8}^{2}$ $-\frac{21}{1280} g_{1}^{2} g_{3}^{2}-\frac{23}{240} g_{1}^{2} g_{2} g_{3}+\frac{11}{3840} g_{1}^{2} g_{2}^{2}+\frac{1285}{768} g_{1}^{3} g_{3}+\frac{117}{640} g_{1}^{3} g_{2}-\frac{248207}{80640} g_{1}^{4}-\frac{4}{15} N_{c} g_{6}^{2} g_{10}^{2}-\frac{2}{15} N_{c} g_{6}^{2} g_{9}^{2}$ $-\frac{8}{15} N_{c} g_{6}^{2} g_{8}^{2}-\frac{2}{15} N_{c} g_{5}^{2} g_{10}^{2}-\frac{1}{15} N_{c} g_{5}^{2} g_{9}^{2}-\frac{4}{15} N_{c} g_{5}^{2} g_{8}^{2}+\frac{13}{240} N_{c} g_{3}^{2} g_{6}^{2}+\frac{1}{120} N_{c} g_{3}^{2} g_{5}^{2}+\frac{9}{40} N_{c} g_{2} g_{3} g_{6}^{2}+\frac{27}{80} N_{c} g_{2}^{2} g_{6}^{2}$ $-\frac{31}{120} N_{c} g_{1} g_{3} g_{6}^{2}+\frac{17}{60} N_{c} g_{1} g_{3} g_{5}^{2}-\frac{293}{120} N_{c} g_{1} g_{2} g_{6}^{2}+\frac{1}{60} N_{c} g_{1} g_{2} g_{5}^{2}+\frac{275}{336} N_{c} g_{1}^{2} g_{6}^{2}+\frac{53}{120} N_{c} g_{1}^{2} g_{5}^{2}-\frac{2}{15} N_{c}^{2} g_{6}^{4}$ $-\frac{2}{15} N_{c}^{2} g_{5}^{2} g_{6}^{2}-\frac{1}{30} N_{c}^{2} g_{5}^{4}+\frac{17}{2520 N_{c}} N_{f} g_{1}^{4}+\frac{1}{35} N_{f} g_{1}^{2} g_{6}^{2}+O\left(g_{i}^{6}\right)$

$\beta_{7}\left(g_{i}\right)=\frac{52}{15 N_{c}^{2}} g_{11}^{4}+\frac{568}{15 N_{c}^{2}} g_{10}^{2} g_{11}^{2}+\frac{1208}{15 N_{c}^{2}} g_{10}^{4}+\frac{56}{15 N_{c}^{2}} g_{9}^{2} g_{11}^{2}+\frac{328}{15 N_{c}^{2}} g_{9}^{2} g_{10}^{2}+\frac{16}{15 N_{c}^{2}} g_{9}^{4}+\frac{16}{15 N_{c}^{2}} g_{8}^{2} g_{11}^{2}+\frac{32}{3 N_{c}^{2}} g_{8}^{2} g_{10}^{2}$ $+\frac{16}{15 N_{c}^{2}} g_{8}^{2} g_{9}^{2}+\frac{32}{15 N_{c}^{2}} g_{8}^{4}+\frac{3}{10 N_{c}^{2}} g_{3}^{2} g_{11}^{2}+\frac{31}{15 N_{c}^{2}} g_{3}^{2} g_{10}^{2}+\frac{1}{6 N_{c}^{2}} g_{3}^{2} g_{9}^{2}+\frac{18}{5 N_{c}^{2}} g_{1} g_{3} g_{11}^{2}+\frac{43}{3 N_{c}^{2}} g_{1} g_{3} g_{10}^{2}+\frac{13}{5 N_{c}^{2}} g_{1} g_{3} g_{9}^{2}$ 
$+\frac{26}{15 N_{c}^{2}} g_{1} g_{3} g_{8}^{2}+\frac{34}{15 N_{c}^{2}} g_{1} g_{2} g_{11}^{2}+\frac{47}{5 N_{c}^{2}} g_{1} g_{2} g_{10}^{2}+\frac{4}{3 N_{c}^{2}} g_{1} g_{2} g_{9}^{2}+\frac{2}{15 N_{c}^{2}} g_{1} g_{2} g_{8}^{2}-\frac{617}{30 N_{c}^{2}} g_{1}^{2} g_{11}^{2}-\frac{78}{N_{c}^{2}} g_{1}^{2} g_{10}^{2}$ $-\frac{331}{30 N_{c}^{2}} g_{1}^{2} g_{9}^{2}-\frac{34}{3 N_{c}^{2}} g_{1}^{2} g_{8}^{2}+\frac{112}{3 N_{c}} g_{7}^{2} g_{11}^{2}+\frac{464}{3 N_{c}} g_{7}^{2} g_{10}^{2}+\frac{64}{3 N_{c}} g_{7}^{2} g_{9}^{2}+\frac{32}{3 N_{c}} g_{7}^{2} g_{8}^{2}+\frac{8}{15 N_{c}} g_{6}^{2} g_{11}^{2}+\frac{16}{3 N_{c}} g_{6}^{2} g_{10}^{2}$ $+\frac{4}{15 N_{c}} g_{6}^{2} g_{9}^{2}+\frac{4}{15 N_{c}} g_{5}^{2} g_{11}^{2}+\frac{8}{3 N_{c}} g_{5}^{2} g_{10}^{2}+\frac{2}{15 N_{c}} g_{5}^{2} g_{9}^{2}+\frac{52}{15 N_{c}} g_{4}^{2} g_{11}^{2}+\frac{284}{15 N_{c}} g_{4}^{2} g_{10}^{2}+\frac{28}{15 N_{c}} g_{4}^{2} g_{9}^{2}+\frac{8}{15 N_{c}} g_{4}^{2} g_{8}^{2}$ $-\frac{31}{30 N_{c}} g_{3}^{2} g_{6}^{2}-\frac{3}{20 N_{c}} g_{3}^{2} g_{5}^{2}-\frac{1}{12 N_{c}} g_{3}^{2} g_{4}^{2}-\frac{13}{15 N_{c}} g_{1} g_{3} g_{7}^{2}-\frac{43}{6 N_{c}} g_{1} g_{3} g_{6}^{2}-\frac{9}{5 N_{c}} g_{1} g_{3} g_{5}^{2}-\frac{13}{10 N_{c}} g_{1} g_{3} g_{4}^{2}-\frac{1}{15 N_{c}} g_{1} g_{2} g_{7}^{2}$ $-\frac{47}{10 N_{c}} g_{1} g_{2} g_{6}^{2}-\frac{17}{15 N_{c}} g_{1} g_{2} g_{5}^{2}-\frac{2}{3 N_{c}} g_{1} g_{2} g_{4}^{2}+\frac{17}{3 N_{c}} g_{1}^{2} g_{7}^{2}+\frac{39}{N_{c}} g_{1}^{2} g_{6}^{2}+\frac{617}{60 N_{c}} g_{1}^{2} g_{5}^{2}+\frac{331}{60 N_{c}} g_{1}^{2} g_{4}^{2}-\frac{13}{15} g_{11}^{4}$ $-\frac{142}{15} g_{10}^{2} g_{11}^{2}-\frac{302}{15} g_{10}^{4}-\frac{14}{15} g_{9}^{2} g_{11}^{2}-\frac{82}{15} g_{9}^{2} g_{10}^{2}-\frac{4}{15} g_{9}^{4}-\frac{4}{15} g_{8}^{2} g_{11}^{2}-\frac{8}{3} g_{8}^{2} g_{10}^{2}-\frac{4}{15} g_{8}^{2} g_{9}^{2}-\frac{8}{15} g_{8}^{4}+\frac{232}{15} g_{7}^{4}$ $-\frac{112}{3} g_{6}^{2} g_{7}^{2}-\frac{22}{15} g_{6}^{4}-\frac{26}{3} g_{5}^{2} g_{7}^{2}-\frac{4}{5} g_{5}^{2} g_{6}^{2}-\frac{1}{15} g_{5}^{4}-\frac{14}{15} g_{4}^{2} g_{7}^{2}-\frac{24}{5} g_{4}^{2} g_{6}^{2}-\frac{13}{15} g_{4}^{2} g_{5}^{2}-\frac{4}{15} g_{4}^{4}-\frac{3}{40} g_{3}^{2} g_{11}^{2}-\frac{31}{60} g_{3}^{2} g_{10}^{2}$ $-\frac{1}{24} g_{3}^{2} g_{9}^{2}-\frac{1}{1920} g_{3}^{4}-\frac{9}{10} g_{1} g_{3} g_{11}^{2}-\frac{43}{12} g_{1} g_{3} g_{10}^{2}-\frac{13}{20} g_{1} g_{3} g_{9}^{2}-\frac{13}{30} g_{1} g_{3} g_{8}^{2}-\frac{53}{1280} g_{1} g_{3}^{3}-\frac{17}{30} g_{1} g_{2} g_{11}^{2}-\frac{47}{20} g_{1} g_{2} g_{10}^{2}$ $-\frac{1}{3} g_{1} g_{2} g_{9}^{2}-\frac{1}{30} g_{1} g_{2} g_{8}^{2}-\frac{5}{384} g_{1} g_{2} g_{3}^{2}-\frac{7}{3840} g_{1} g_{2}^{2} g_{3}+\frac{617}{120} g_{1}^{2} g_{11}^{2}+\frac{39}{2} g_{1}^{2} g_{10}^{2}+\frac{331}{120} g_{1}^{2} g_{9}^{2}+\frac{17}{6} g_{1}^{2} g_{8}^{2}+\frac{31}{3840} g_{1}^{2} g_{3}^{2}$ $-\frac{47}{120} g_{1}^{2} g_{2} g_{3}-\frac{169}{1280} g_{1}^{2} g_{2}^{2}+\frac{7423}{3840} g_{1}^{3} g_{3}+\frac{3673}{1920} g_{1}^{3} g_{2}-\frac{157663}{26880} g_{1}^{4}-\frac{28}{3} N_{c} g_{7}^{2} g_{11}^{2}-\frac{116}{3} N_{c} g_{7}^{2} g_{10}^{2}-\frac{16}{3} N_{c} g_{7}^{2} g_{9}^{2}$ $-\frac{8}{3} N_{c} g_{7}^{2} g_{8}^{2}-\frac{2}{15} N_{c} g_{6}^{2} g_{11}^{2}-\frac{4}{3} N_{c} g_{6}^{2} g_{10}^{2}-\frac{1}{15} N_{c} g_{6}^{2} g_{9}^{2}-\frac{1}{15} N_{c} g_{5}^{2} g_{11}^{2}-\frac{2}{3} N_{c} g_{5}^{2} g_{10}^{2}-\frac{1}{30} N_{c} g_{5}^{2} g_{9}^{2}-\frac{13}{15} N_{c} g_{4}^{2} g_{11}^{2}$ $-\frac{71}{15} N_{c} g_{4}^{2} g_{10}^{2}-\frac{7}{15} N_{c} g_{4}^{2} g_{9}^{2}-\frac{2}{15} N_{c} g_{4}^{2} g_{8}^{2}-\frac{37}{80} N_{c} g_{3}^{2} g_{7}^{2}-\frac{3}{80} N_{c} g_{3}^{2} g_{4}^{2}+\frac{9}{40} N_{c} g_{2} g_{3} g_{7}^{2}+\frac{27}{80} N_{c} g_{2}^{2} g_{7}^{2}-\frac{153}{40} N_{c} g_{1} g_{3} g_{7}^{2}$ $-\frac{7}{60} N_{c} g_{1} g_{3} g_{6}^{2}-\frac{7}{120} N_{c} g_{1} g_{3} g_{5}^{2}-\frac{9}{20} N_{c} g_{1} g_{3} g_{4}^{2}-\frac{557}{120} N_{c} g_{1} g_{2} g_{7}^{2}-\frac{1}{12} N_{c} g_{1} g_{2} g_{6}^{2}-\frac{1}{24} N_{c} g_{1} g_{2} g_{5}^{2}-\frac{17}{60} N_{c} g_{1} g_{2} g_{4}^{2}$ $+\frac{11257}{560} N_{c} g_{1}^{2} g_{7}^{2}+\frac{49}{60} N_{c} g_{1}^{2} g_{6}^{2}+\frac{49}{120} N_{c} g_{1}^{2} g_{5}^{2}+\frac{617}{240} N_{c} g_{1}^{2} g_{4}^{2}-\frac{56}{3} N_{c}^{2} g_{7}^{4}-\frac{4}{3} N_{c}^{2} g_{6}^{2} g_{7}^{2}-\frac{2}{3} N_{c}^{2} g_{5}^{2} g_{7}^{2}-\frac{14}{3} N_{c}^{2} g_{4}^{2} g_{7}^{2}$ $-\frac{1}{15} N_{c}^{2} g_{4}^{2} g_{6}^{2}-\frac{1}{30} N_{c}^{2} g_{4}^{2} g_{5}^{2}-\frac{13}{60} N_{c}^{2} g_{4}^{4}+\frac{1}{5040 N_{c}} N_{f} g_{1}^{4}+\frac{1}{35} N_{f} g_{1}^{2} g_{7}^{2}+O\left(g_{i}^{6}\right)$ $\beta_{8}\left(g_{i}\right)=\frac{58}{15 N_{c}} g_{11}^{4}+\frac{128}{3 N_{c}} g_{10}^{2} g_{11}^{2}+\frac{1328}{15 N_{c}} g_{10}^{4}+\frac{139}{15 N_{c}} g_{9}^{2} g_{11}^{2}+\frac{764}{15 N_{c}} g_{9}^{2} g_{10}^{2}+\frac{71}{15 N_{c}} g_{9}^{4}+\frac{896}{15 N_{c}} g_{8}^{2} g_{11}^{2}+\frac{248 N_{c}}{g} g_{10}^{2}$ $+\frac{788}{15 N_{c}} g_{8}^{2} g_{9}^{2}+\frac{464}{5 N_{c}} g_{8}^{4}+\frac{3}{20 N_{c}} g_{3}^{2} g_{11}^{2}+\frac{31}{30 N_{c}} g_{3}^{2} g_{10}^{2}+\frac{1}{12 N_{c}} g_{3}^{2} g_{9}^{2}+\frac{9}{5 N_{c}} g_{1} g_{3} g_{11}^{2}+\frac{43}{6 N_{c}} g_{1} g_{3} g_{10}^{2}+\frac{13}{10 N_{c}} g_{1} g_{3} g_{9}^{2}$ $+\frac{13}{15 N_{c}} g_{1} g_{3} g_{8}^{2}+\frac{17}{15 N_{c}} g_{1} g_{2} g_{11}^{2}+\frac{47}{10 N_{c}} g_{1} g_{2} g_{10}^{2}+\frac{2}{3 N_{c}} g_{1} g_{2} g_{9}^{2}+\frac{1}{15 N_{c}} g_{1} g_{2} g_{8}^{2}-\frac{617}{60 N_{c}} g_{1}^{2} g_{11}^{2}-\frac{39}{N_{c}} g_{1}^{2} g_{10}^{2}$ $-\frac{331}{60 N_{c}} g_{1}^{2} g_{9}^{2}-\frac{17}{3 N_{c}} g_{1}^{2} g_{8}^{2}-\frac{4}{15} g_{7}^{2} g_{9}^{2}-\frac{56}{15} g_{7}^{2} g_{8}^{2}-\frac{2}{3} g_{6}^{2} g_{11}^{2}-\frac{8}{5} g_{6}^{2} g_{10}^{2}-\frac{24}{5} g_{6}^{2} g_{9}^{2}-\frac{116}{3} g_{6}^{2} g_{8}^{2}-\frac{1}{15} g_{5}^{2} g_{11}^{2}-\frac{2}{15} g_{5}^{2} g_{10}^{2}$ $-\frac{13}{15} g_{5}^{2} g_{9}^{2}-\frac{28}{3} g_{5}^{2} g_{8}^{2}-\frac{1}{30} g_{4}^{2} g_{11}^{2}-\frac{2}{15} g_{4}^{2} g_{10}^{2}-\frac{1}{2} g_{4}^{2} g_{9}^{2}-\frac{82}{15} g_{4}^{2} g_{8}^{2}-\frac{31}{60} g_{3}^{2} g_{6}^{2}-\frac{3}{40} g_{3}^{2} g_{5}^{2}-\frac{1}{24} g_{3}^{2} g_{4}^{2}-\frac{13}{30} g_{1} g_{3} g_{7}^{2}$ $-\frac{43}{12} g_{1} g_{3} g_{6}^{2}-\frac{9}{10} g_{1} g_{3} g_{5}^{2}-\frac{13}{20} g_{1} g_{3} g_{4}^{2}-\frac{1}{30} g_{1} g_{2} g_{7}^{2}-\frac{47}{20} g_{1} g_{2} g_{6}^{2}-\frac{17}{30} g_{1} g_{2} g_{5}^{2}-\frac{1}{3} g_{1} g_{2} g_{4}^{2}+\frac{17}{6} g_{1}^{2} g_{7}^{2}+\frac{39}{2} g_{1}^{2} g_{6}^{2}$ $+\frac{617}{120} g_{1}^{2} g_{5}^{2}+\frac{331}{120} g_{1}^{2} g_{4}^{2}-\frac{1}{4} N_{c} g_{11}^{4}-\frac{83}{30} N_{c} g_{10}^{2} g_{11}^{2}-\frac{57}{10} N_{c} g_{10}^{4}-\frac{43}{60} N_{c} g_{9}^{2} g_{11}^{2}-\frac{229}{60} N_{c} g_{9}^{2} g_{10}^{2}-\frac{41}{80} N_{c} g_{9}^{4}$ $-\frac{27}{5} N_{c} g_{8}^{2} g_{11}^{2}-\frac{106}{5} N_{c} g_{8}^{2} g_{10}^{2}-\frac{223}{30} N_{c} g_{8}^{2} g_{9}^{2}-\frac{302}{15} N_{c} g_{8}^{4}-\frac{3}{160} N_{c} g_{3}^{2} g_{11}^{2}-\frac{31}{240} N_{c} g_{3}^{2} g_{10}^{2}-\frac{9}{320} N_{c} g_{3}^{2} g_{9}^{2}$ 
$-\frac{49}{240} N_{c} g_{3}^{2} g_{8}^{2}-\frac{1}{7680} N_{c} g_{3}^{4}+\frac{9}{40} N_{c} g_{2} g_{3} g_{8}^{2}+\frac{27}{80} N_{c} g_{2}^{2} g_{8}^{2}-\frac{61}{240} N_{c} g_{1} g_{3} g_{11}^{2}-\frac{223}{240} N_{c} g_{1} g_{3} g_{10}^{2}-\frac{169}{480} N_{c} g_{1} g_{3} g_{9}^{2}$

$-\frac{11}{5} N_{c} g_{1} g_{3} g_{8}^{2}-\frac{13}{1536} N_{c} g_{1} g_{3}^{3}-\frac{13}{80} N_{c} g_{1} g_{2} g_{11}^{2}-\frac{31}{48} N_{c} g_{1} g_{2} g_{10}^{2}-\frac{107}{480} N_{c} g_{1} g_{2} g_{9}^{2}-\frac{211}{60} N_{c} g_{1} g_{2} g_{8}^{2}$

$+\frac{7}{3840} N_{c} g_{1} g_{2} g_{3}^{2}-\frac{7}{7680} N_{c} g_{1} g_{2}^{2} g_{3}+\frac{143}{96} N_{c} g_{1}^{2} g_{11}^{2}+\frac{77}{15} N_{c} g_{1}^{2} g_{10}^{2}+\frac{1949}{960} N_{c} g_{1}^{2} g_{9}^{2}+\frac{19267}{1680} N_{c} g_{1}^{2} g_{8}^{2}$

$+\frac{13}{2560} N_{c} g_{1}^{2} g_{3}^{2}-\frac{259}{1920} N_{c} g_{1}^{2} g_{2} g_{3}-\frac{383}{7680} N_{c} g_{1}^{2} g_{2}^{2}+\frac{3961}{7680} N_{c} g_{1}^{3} g_{3}+\frac{889}{1280} N_{c} g_{1}^{3} g_{2}-\frac{29269}{16128} N_{c} g_{1}^{4}+\frac{1}{35} N_{f} g_{1}^{2} g_{8}^{2}$

$+\frac{1}{10080} N_{f} g_{1}^{4}+O\left(g_{i}^{6}\right)$

$\beta_{9}\left(g_{i}\right)=\frac{70}{3 N_{c}} g_{11}^{4}+\frac{116}{5 N_{c}} g_{10}^{2} g_{11}^{2}+\frac{56}{15 N_{c}} g_{10}^{4}+\frac{34 N_{c}^{2}}{g} g_{11}^{2}+\frac{92}{5 N_{c}} g_{9}^{2} g_{10}^{2}+\frac{58}{3 N_{c}} g_{9}^{4}+\frac{232}{15 N_{c}} g_{8}^{2} g_{11}^{2}+\frac{112}{15 N_{c}} g_{8}^{2} g_{10}^{2}+\frac{368}{15 N_{c}} g_{8}^{2} g_{9}^{2}$

$+\frac{32}{5 N_{c}} g_{8}^{4}-\frac{1}{60 N_{c}} g_{3}^{2} g_{11}^{2}+\frac{1}{30 N_{c}} g_{3}^{2} g_{10}^{2}+\frac{1}{2 N_{c}} g_{3}^{2} g_{9}^{2}+\frac{2}{3 N_{c}} g_{3}^{2} g_{8}^{2}+\frac{1}{30 N_{c}} g_{1} g_{3} g_{11}^{2}+\frac{22}{15 N_{c}} g_{1} g_{3} g_{10}^{2}-\frac{17}{30 N_{c}} g_{1} g_{3} g_{9}^{2}$

$+\frac{34}{15 N_{c}} g_{1} g_{3} g_{8}^{2}+\frac{5}{6 N_{c}} g_{1} g_{2} g_{11}^{2}+\frac{4}{15 N_{c}} g_{1} g_{2} g_{10}^{2}-\frac{3}{5 N_{c}} g_{1} g_{2} g_{9}^{2}-\frac{2}{5 N_{c}} g_{1} g_{2} g_{8}^{2}-\frac{41}{60 N_{c}} g_{1}^{2} g_{11}^{2}+\frac{31}{30 N_{c}} g_{1}^{2} g_{10}^{2}$

$+\frac{13}{5 N_{c}} g_{1}^{2} g_{9}^{2}+\frac{48}{5 N_{c}} g_{1}^{2} g_{8}^{2}-\frac{8}{15} g_{7}^{2} g_{11}^{2}-\frac{16}{15} g_{7}^{2} g_{10}^{2}-\frac{52}{15} g_{7}^{2} g_{9}^{2}-\frac{32}{15} g_{7}^{2} g_{8}^{2}-\frac{8}{5} g_{6}^{2} g_{11}^{2}-\frac{4}{5} g_{6}^{2} g_{10}^{2}-\frac{16}{15} g_{6}^{2} g_{9}^{2}-\frac{8}{15} g_{6}^{2} g_{8}^{2}$

$-\frac{26}{5} g_{5}^{2} g_{11}^{2}-\frac{46}{15} g_{5}^{2} g_{10}^{2}-3 g_{5}^{2} g_{9}^{2}-\frac{4}{15} g_{5}^{2} g_{8}^{2}-\frac{16}{15} g_{4}^{2} g_{11}^{2}-\frac{6}{5} g_{4}^{2} g_{10}^{2}-\frac{16}{5} g_{4}^{2} g_{9}^{2}-\frac{28}{15} g_{4}^{2} g_{8}^{2}-\frac{1}{3} g_{3}^{2} g_{7}^{2}-\frac{1}{60} g_{3}^{2} g_{6}^{2}$

$+\frac{1}{120} g_{3}^{2} g_{5}^{2}-\frac{1}{4} g_{3}^{2} g_{4}^{2}-\frac{17}{15} g_{1} g_{3} g_{7}^{2}-\frac{11}{15} g_{1} g_{3} g_{6}^{2}-\frac{1}{60} g_{1} g_{3} g_{5}^{2}+\frac{17}{60} g_{1} g_{3} g_{4}^{2}+\frac{1}{5} g_{1} g_{2} g_{7}^{2}-\frac{2}{15} g_{1} g_{2} g_{6}^{2}-\frac{5}{12} g_{1} g_{2} g_{5}^{2}$

$+\frac{3}{10} g_{1} g_{2} g_{4}^{2}-\frac{24}{5} g_{1}^{2} g_{7}^{2}-\frac{31}{60} g_{1}^{2} g_{6}^{2}+\frac{41}{120} g_{1}^{2} g_{5}^{2}-\frac{13}{10} g_{1}^{2} g_{4}^{2}-\frac{23}{15} N_{c} g_{11}^{4}-\frac{26}{15} N_{c} g_{10}^{2} g_{11}^{2}-\frac{1}{3} N_{c} g_{10}^{4}-\frac{32}{15} N_{c} g_{9}^{2} g_{11}^{2}$

$-\frac{26}{15} N_{c} g_{9}^{2} g_{10}^{2}-\frac{107}{60} N_{c} g_{9}^{4}-\frac{4}{15} N_{c} g_{8}^{2} g_{11}^{2}-\frac{4}{15} N_{c} g_{8}^{2} g_{10}^{2}-\frac{8}{5} N_{c} g_{8}^{2} g_{9}^{2}-\frac{4}{15} N_{c} g_{8}^{4}-\frac{1}{30} N_{c} g_{3}^{2} g_{11}^{2}-\frac{3}{40} N_{c} g_{3}^{2} g_{10}^{2}$

$-\frac{11}{60} N_{c} g_{3}^{2} g_{9}^{2}-\frac{3}{20} N_{c} g_{3}^{2} g_{8}^{2}-\frac{7}{1280} N_{c} g_{3}^{4}+\frac{9}{40} N_{c} g_{2} g_{3} g_{9}^{2}+\frac{27}{80} N_{c} g_{2}^{2} g_{9}^{2}-\frac{1}{20} N_{c} g_{1} g_{3} g_{11}^{2}-\frac{1}{6} N_{c} g_{1} g_{3} g_{10}^{2}$

$-\frac{29}{120} N_{c} g_{1} g_{3} g_{9}^{2}+\frac{13}{1920} N_{c} g_{1} g_{3}^{3}-\frac{1}{5} N_{c} g_{1} g_{2} g_{11}^{2}-\frac{1}{30} N_{c} g_{1} g_{2} g_{10}^{2}-\frac{34}{15} N_{c} g_{1} g_{2} g_{9}^{2}+\frac{2}{15} N_{c} g_{1} g_{2} g_{8}^{2}+\frac{1}{240} N_{c} g_{1} g_{2} g_{3}^{2}$

$-\frac{1}{1920} N_{c} g_{1} g_{2}^{2} g_{3}-\frac{7}{60} N_{c} g_{1}^{2} g_{11}^{2}-\frac{47}{120} N_{c} g_{1}^{2} g_{10}^{2}-\frac{583}{840} N_{c} g_{1}^{2} g_{9}^{2}-\frac{91}{60} N_{c} g_{1}^{2} g_{8}^{2}+\frac{3}{320} N_{c} g_{1}^{2} g_{3}^{2}+\frac{59}{320} N_{c} g_{1}^{2} g_{2} g_{3}$

$+\frac{331}{1920} N_{c} g_{1}^{2} g_{2}^{2}-\frac{161}{384} N_{c} g_{1}^{3} g_{3}-\frac{331}{320} N_{c} g_{1}^{3} g_{2}+\frac{87677}{80640} N_{c} g_{1}^{4}+\frac{1}{35} N_{f} g_{1}^{2} g_{9}^{2}+\frac{5}{252} N_{f} g_{1}^{4}+O\left(g_{i}^{6}\right)$

$\beta_{10}\left(g_{i}\right)=\frac{18}{5 N_{c}} g_{11}^{4}+\frac{196}{5 N_{c}} g_{10}^{2} g_{11}^{2}+\frac{248}{3 N_{c}} g_{10}^{4}+\frac{62}{15 N_{c}} g_{9}^{2} g_{11}^{2}+\frac{116}{5 N_{c}} g_{9}^{2} g_{10}^{2}+\frac{6}{5 N_{c}} g_{9}^{4}+\frac{8}{3 N_{c}} g_{8}^{2} g_{11}^{2}+\frac{272}{15 N_{c}} g_{8}^{2} g_{10}^{2}+\frac{32}{15 N_{c}} g_{8}^{2} g_{9}^{2}$

$+\frac{32}{15 N_{c}} g_{8}^{4}-\frac{3}{20 N_{c}} g_{3}^{2} g_{11}^{2}-\frac{31}{30 N_{c}} g_{3}^{2} g_{10}^{2}-\frac{1}{12 N_{c}} g_{3}^{2} g_{9}^{2}-\frac{9}{5 N_{c}} g_{1} g_{3} g_{11}^{2}-\frac{43}{6 N_{c}} g_{1} g_{3} g_{10}^{2}-\frac{13}{10 N_{c}} g_{1} g_{3} g_{9}^{2}-\frac{13}{15 N_{c}} g_{1} g_{3} g_{8}^{2}$

$-\frac{17}{15 N_{c}} g_{1} g_{2} g_{11}^{2}-\frac{47}{10 N_{c}} g_{1} g_{2} g_{10}^{2}-\frac{2}{3 N_{c}} g_{1} g_{2} g_{9}^{2}-\frac{1}{15 N_{c}} g_{1} g_{2} g_{8}^{2}+\frac{617}{60 N_{c}} g_{1}^{2} g_{11}^{2}+\frac{39}{N_{c}} g_{1}^{2} g_{10}^{2}+\frac{331}{60 N_{c}} g_{1}^{2} g_{9}^{2}+\frac{17}{3 N_{c}} g_{1}^{2} g_{8}^{2}$

$-\frac{8}{15} g_{7}^{2} g_{11}^{2}-\frac{56}{15} g_{7}^{2} g_{10}^{2}-\frac{4}{15} g_{7}^{2} g_{9}^{2}-\frac{48}{5} g_{6}^{2} g_{11}^{2}-\frac{604}{15} g_{6}^{2} g_{10}^{2}-\frac{82}{15} g_{6}^{2} g_{9}^{2}-\frac{16}{5} g_{6}^{2} g_{8}^{2}-\frac{26}{15} g_{5}^{2} g_{11}^{2}-\frac{142}{15} g_{5}^{2} g_{10}^{2}-\frac{14}{15} g_{5}^{2} g_{9}^{2}$

$-\frac{4}{15} g_{5}^{2} g_{8}^{2}-g_{4}^{2} g_{11}^{2}-\frac{28}{5} g_{4}^{2} g_{10}^{2}-\frac{8}{15} g_{4}^{2} g_{9}^{2}-\frac{4}{15} g_{4}^{2} g_{8}^{2}+\frac{31}{60} g_{3}^{2} g_{6}^{2}+\frac{3}{40} g_{3}^{2} g_{5}^{2}+\frac{1}{24} g_{3}^{2} g_{4}^{2}+\frac{13}{30} g_{1} g_{3} g_{7}^{2}+\frac{43}{12} g_{1} g_{3} g_{6}^{2}$

$+\frac{9}{10} g_{1} g_{3} g_{5}^{2}+\frac{13}{20} g_{1} g_{3} g_{4}^{2}+\frac{1}{30} g_{1} g_{2} g_{7}^{2}+\frac{47}{20} g_{1} g_{2} g_{6}^{2}+\frac{17}{30} g_{1} g_{2} g_{5}^{2}+\frac{1}{3} g_{1} g_{2} g_{4}^{2}-\frac{17}{6} g_{1}^{2} g_{7}^{2}-\frac{39}{2} g_{1}^{2} g_{6}^{2}-\frac{617}{120} g_{1}^{2} g_{5}^{2}$

$-\frac{331}{120} g_{1}^{2} g_{4}^{2}-\frac{1}{30} N_{c} g_{11}^{4}-\frac{1}{5} N_{c} g_{10}^{2} g_{11}^{2}-\frac{4}{15} N_{c} g_{10}^{4}-\frac{1}{30} N_{c} g_{9}^{2} g_{11}^{2}-\frac{1}{10} N_{c} g_{9}^{2} g_{10}^{2}-\frac{1}{120} N_{c} g_{9}^{4}-\frac{2}{15} N_{c} g_{8}^{2} g_{11}^{2}$ 


$$
\begin{aligned}
& -\frac{8}{15} N_{c} g_{8}^{2} g_{10}^{2}-\frac{1}{15} N_{c} g_{8}^{2} g_{9}^{2}+\frac{1}{240} N_{c} g_{3}^{2} g_{11}^{2}+\frac{13}{240} N_{c} g_{3}^{2} g_{10}^{2}+\frac{1}{480} N_{c} g_{3}^{2} g_{9}^{2}+\frac{9}{40} N_{c} g_{2} g_{3} g_{10}^{2}+\frac{27}{80} N_{c} g_{2}^{2} g_{10}^{2} \\
& +\frac{17}{120} N_{c} g_{1} g_{3} g_{11}^{2}-\frac{37}{120} N_{c} g_{1} g_{3} g_{10}^{2}+\frac{17}{240} N_{c} g_{1} g_{3} g_{9}^{2}+\frac{1}{10} N_{c} g_{1} g_{3} g_{8}^{2}-\frac{29}{7680} N_{c} g_{1} g_{3}^{3}+\frac{1}{120} N_{c} g_{1} g_{2} g_{11}^{2} \\
& -\frac{289}{120} N_{c} g_{1} g_{2} g_{10}^{2}+\frac{1}{240} N_{c} g_{1} g_{2} g_{9}^{2}-\frac{1}{15} N_{c} g_{1} g_{2} g_{8}^{2}-\frac{13}{1280} N_{c} g_{1} g_{2} g_{3}^{2}+\frac{7}{7680} N_{c} g_{1} g_{2}^{2} g_{3}+\frac{53}{240} N_{c} g_{1}^{2} g_{11}^{2} \\
& +\frac{1879}{1680} N_{c} g_{1}^{2} g_{10}^{2}+\frac{53}{480} N_{c} g_{1}^{2} g_{9}^{2}-\frac{3}{5} N_{c} g_{1}^{2} g_{8}^{2}-\frac{47}{7680} N_{c} g_{1}^{2} g_{3}^{2}+\frac{71}{960} N_{c} g_{1}^{2} g_{2} g_{3}+\frac{259}{7680} N_{c} g_{1}^{2} g_{2}^{2}-\frac{499}{7680} N_{c} g_{1}^{3} g_{3} \\
& -\frac{1661}{3840} N_{c} g_{1}^{3} g_{2}+\frac{112391}{161280} N_{c} g_{1}^{4}+\frac{1}{35} N_{f} g_{1}^{2} g_{10}^{2}+\frac{17}{5040} N_{f} g_{1}^{4}+O\left(g_{i}^{6}\right) \\
& \beta_{11}\left(g_{i}\right)=\frac{14}{15 N_{c}} g_{11}^{4}+\frac{28}{15 N_{c}} g_{10}^{2} g_{11}^{2}+\frac{8}{3 N_{c}} g_{10}^{4}+\frac{54}{5 N_{c}} g_{9}^{2} g_{11}^{2}+\frac{68}{15 N_{c}} g_{9}^{2} g_{10}^{2}+\frac{6}{5 N_{c}} g_{9}^{4}+\frac{40}{3 N_{c}} g_{8}^{2} g_{11}^{2}+\frac{112}{15 N_{c}} g_{8}^{2} g_{10}^{2} \\
& +\frac{32}{15 N_{c}} g_{8}^{2} g_{9}^{2}+\frac{32}{15 N_{c}} g_{8}^{4}+\frac{1}{60 N_{c}} g_{3}^{2} g_{11}^{2}-\frac{1}{30 N_{c}} g_{3}^{2} g_{10}^{2}-\frac{1}{2 N_{c}} g_{3}^{2} g_{9}^{2}-\frac{2}{3 N_{c}} g_{3}^{2} g_{8}^{2}-\frac{1}{30 N_{c}} g_{1} g_{3} g_{11}^{2}-\frac{22}{15 N_{c}} g_{1} g_{3} g_{10}^{2} \\
& +\frac{17}{30 N_{c}} g_{1} g_{3} g_{9}^{2}-\frac{34}{15 N_{c}} g_{1} g_{3} g_{8}^{2}-\frac{5}{6 N_{c}} g_{1} g_{2} g_{11}^{2}-\frac{4}{15 N_{c}} g_{1} g_{2} g_{10}^{2}+\frac{3}{5 N_{c}} g_{1} g_{2} g_{9}^{2}+\frac{2}{5 N_{c}} g_{1} g_{2} g_{8}^{2}+\frac{41}{60 N_{c}} g_{1}^{2} g_{11}^{2} \\
& -\frac{31}{30 N_{c}} g_{1}^{2} g_{10}^{2}-\frac{13}{5 N_{c}} g_{1}^{2} g_{9}^{2}-\frac{48}{5 N_{c}} g_{1}^{2} g_{8}^{2}-\frac{16}{5} g_{7}^{2} g_{11}^{2}-\frac{16}{15} g_{7}^{2} g_{10}^{2}-\frac{4}{15} g_{7}^{2} g_{9}^{2}-\frac{4}{15} g_{6}^{2} g_{11}^{2}-\frac{4}{15} g_{6}^{2} g_{10}^{2}-\frac{4}{5} g_{6}^{2} g_{9}^{2}-\frac{8}{15} g_{6}^{2} g_{8}^{2} \\
& -\frac{2}{5} g_{5}^{2} g_{11}^{2}-\frac{2}{15} g_{5}^{2} g_{10}^{2}-\frac{13}{5} g_{5}^{2} g_{9}^{2}-\frac{44}{15} g_{5}^{2} g_{8}^{2}-\frac{8}{3} g_{4}^{2} g_{11}^{2}-\frac{14}{15} g_{4}^{2} g_{10}^{2}-\frac{8}{15} g_{4}^{2} g_{9}^{2}-\frac{4}{15} g_{4}^{2} g_{8}^{2}+\frac{1}{3} g_{3}^{2} g_{7}^{2}+\frac{1}{60} g_{3}^{2} g_{6}^{2}-\frac{1}{120} g_{3}^{2} g_{5}^{2} \\
& +\frac{1}{4} g_{3}^{2} g_{4}^{2}+\frac{17}{15} g_{1} g_{3} g_{7}^{2}+\frac{11}{15} g_{1} g_{3} g_{6}^{2}+\frac{1}{60} g_{1} g_{3} g_{5}^{2}-\frac{17}{60} g_{1} g_{3} g_{4}^{2}-\frac{1}{5} g_{1} g_{2} g_{7}^{2}+\frac{2}{15} g_{1} g_{2} g_{6}^{2}+\frac{5}{12} g_{1} g_{2} g_{5}^{2}-\frac{3}{10} g_{1} g_{2} g_{4}^{2} \\
& +\frac{24}{5} g_{1}^{2} g_{7}^{2}+\frac{31}{60} g_{1}^{2} g_{6}^{2}-\frac{41}{120} g_{1}^{2} g_{5}^{2}+\frac{13}{10} g_{1}^{2} g_{4}^{2}+\frac{7}{15} N_{c} g_{11}^{4}+\frac{2}{15} N_{c} g_{10}^{2} g_{11}^{2}-\frac{4}{15} N_{c} g_{10}^{4}-\frac{8}{15} N_{c} g_{9}^{2} g_{11}^{2}-\frac{4}{15} N_{c} g_{9}^{2} g_{10}^{2} \\
& +\frac{7}{60} N_{c} g_{9}^{4}-\frac{4}{5} N_{c} g_{8}^{2} g_{11}^{2}-\frac{8}{15} N_{c} g_{8}^{2} g_{10}^{2}+\frac{4}{15} N_{c} g_{8}^{2} g_{9}^{2}-\frac{1}{16} N_{c} g_{3}^{2} g_{11}^{2}-\frac{1}{40} N_{c} g_{3}^{2} g_{10}^{2}+\frac{13}{240} N_{c} g_{3}^{2} g_{9}^{2}+\frac{1}{12} N_{c} g_{3}^{2} g_{8}^{2} \\
& +\frac{1}{384} N_{c} g_{3}^{4}+\frac{9}{40} N_{c} g_{2} g_{3} g_{11}^{2}+\frac{27}{80} N_{c} g_{2}^{2} g_{11}^{2}-\frac{3}{8} N_{c} g_{1} g_{3} g_{11}^{2}+\frac{7}{15} N_{c} g_{1} g_{3} g_{10}^{2}-\frac{1}{12} N_{c} g_{1} g_{3} g_{9}^{2}+\frac{1}{5} N_{c} g_{1} g_{3} g_{8}^{2} \\
& +\frac{1}{384} N_{c} g_{1} g_{3}^{3}-\frac{53}{24} N_{c} g_{1} g_{2} g_{11}^{2}+\frac{1}{12} N_{c} g_{1} g_{2} g_{10}^{2}-\frac{1}{8} N_{c} g_{1} g_{2} g_{9}^{2}-\frac{1}{10} N_{c} g_{1} g_{2} g_{8}^{2}+\frac{1}{1920} N_{c} g_{1} g_{2}^{2} g_{3}-\frac{361}{1680} N_{c} g_{1}^{2} g_{11}^{2} \\
& +\frac{23}{120} N_{c} g_{1}^{2} g_{10}^{2}+\frac{71}{240} N_{c} g_{1}^{2} g_{9}^{2}+\frac{83}{60} N_{c} g_{1}^{2} g_{8}^{2}+\frac{13}{1920} N_{c} g_{1}^{2} g_{3}^{2}-\frac{63}{320} N_{c} g_{1}^{2} g_{2} g_{3}-\frac{111}{640} N_{c} g_{1}^{2} g_{2}^{2}+\frac{631}{1920} N_{c} g_{1}^{3} g_{3} \\
& +\frac{1001}{960} N_{c} g_{1}^{3} g_{2}-\frac{23629}{20160} N_{c} g_{1}^{4}+\frac{1}{35} N_{f} g_{1}^{2} g_{11}^{2}-\frac{149}{5040} N_{f} g_{1}^{4}+O\left(g_{i}^{6}\right) \text {. }
\end{aligned}
$$

Again these renormalization group functions, as well as those for $S U(3)$, satisfy the same checks we discussed for the $S U(2)$ case.

\section{LARGE $N_{f}$ CHECK}

We devote this section to the final independent check we have on the renormalization group functions in each of the three cases which is the comparison with the large $N_{f}$ critical exponents which have been computed in the nonAbelian Thirring model universality class. The background to this is the observation that the renormalization group functions depend on the parameter $N_{f}$ and the various coupling constants for a specific value of $N_{c}$. The coefficients of these parameters in each renormalization group function is conventionally determined by perturbative methods as was carried out in the previous section. However one can also determine the coefficients via an ordering of graphs defined by $N_{f}$. This is achieved through the known $d$-dependent critical exponents of the underlying universality class. An alternative view of this is that the exponents already contain information on the perturbative coefficients. The method is to compute the renormalization group functions at the Wilson-Fisher fixed point in $d=8-2 \epsilon$, expand in powers of $1 / N_{f}$ and then compare with the $\epsilon$ expansion of the corresponding large $N_{f}$ critical 
exponents. This constitutes our independent check. The first step in the procedure is to locate the Wilson-Fisher fixed point explicitly order by order in powers of $1 / N_{f}$ and $\epsilon$ by finding the solution to

$$
\beta_{i}\left(g_{j}\right)=0
$$

for the $d$-dimensional $\beta$-functions. In four dimensions this is relatively straightforward since there is only one coupling constant in QCD. For eight dimensions we have 11 coupling constants for the case of $S U\left(N_{c}\right)$. So we follow the method introduced in $[22,23]$. As there are 3- and 4-leg operators in (2.8) we have to be careful in defining the rescaling which is the initial step in the approach of $[22,23]$. Therefore at the outset we set

$$
\begin{aligned}
& g_{i}=\sqrt{\frac{70 \epsilon}{N_{f}}} x_{i} \quad i=1 \text { to } 3 \\
& g_{i}^{2}=\frac{70 \epsilon}{N_{f}} x_{i} \quad i=4 \text { to } 11
\end{aligned}
$$

in (5.1) and expand in powers of $\epsilon$ and $1 / N_{f}$. First the leading order term in $1 / N_{f}$ of the equations is isolated and then the $\epsilon$ expansion of this leading term is found before repeating the exercise for the subsequent term in the large $N_{f}$ expansion. For the $S U\left(N_{c}\right) \beta$-functions the resulting critical couplings are

$$
\begin{aligned}
x_{1} & =1+\frac{1933 N_{c}}{24 N_{f}}+\frac{3736489 N_{c}^{2}}{384 N_{f}^{2}}+O\left(\epsilon ; \frac{1}{N_{f}^{3}}\right) \\
x_{2} & =\frac{17}{9}+\frac{287279 N_{c}}{1944 N_{f}}+\frac{5066611513 N_{c}^{2}}{279936 N_{f}^{2}}+O\left(\epsilon ; \frac{1}{N_{f}^{3}}\right) \\
x_{3} & =\frac{16}{3}+\frac{143411 N_{c}}{324 N_{f}}+\frac{153781987 N_{c}^{2}}{2916 N_{f}^{2}}+O\left(\epsilon ; \frac{1}{N_{f}^{3}}\right) \\
x_{4}= & -\frac{25}{9 N_{c}}-\left[\frac{1615081}{46656}+\frac{115591}{432 N_{c}^{2}}\right] \frac{1}{N_{f}}+\left[\frac{4084305085}{11664 N_{c}^{3}}-\frac{318375286621}{839808 N_{c}}+\frac{894758019623 N_{c}}{3359232}\right] \frac{1}{N_{f}^{2}}+O\left(\epsilon ; \frac{1}{N_{f}^{3}}\right) \\
x_{5}= & \frac{149}{36 N_{c}}+\left[\frac{39472453}{46656}-\frac{343}{432 N_{c}^{2}}\right] \frac{1}{N_{f}}+\left[-\frac{768922651}{11664 N_{c}^{3}}+\frac{6468807373}{839808 N_{c}}+\frac{144625900963 N_{c}}{1119744}\right] \frac{1}{N_{f}^{2}}+O\left(\epsilon ; \frac{1}{N_{f}^{3}}\right) \\
x_{6}= & \frac{149}{72}+\left[\frac{18279803 N_{c}}{46656}-\frac{343}{432 N_{c}}\right] \frac{1}{N_{f}}+\left[\frac{161927831371 N_{c}^{2}}{2239488}-\frac{28392695975}{1679616}-\frac{1571677793}{46656 N_{c}^{2}}\right] \frac{1}{N_{f}^{2}}+O\left(\epsilon ; \frac{1}{N_{f}^{3}}\right) \\
x_{7}= & -\frac{17}{36 N_{c}}-\left[\frac{997943}{46656}+\frac{343}{432 N_{c}^{2}}\right] \frac{1}{N_{f}}+\left[\frac{285596549}{2916 N_{c}^{3}}+\frac{155421633577}{839808 N_{c}}+\frac{69408246905 N_{c}}{1119744}\right] \frac{1}{N_{f}^{2}}+O\left(\epsilon ; \frac{1}{N_{f}^{3}}\right) \\
x_{8}= & -\frac{1}{72 N_{c}}+\left[\frac{343}{216 N_{c}^{2}}-\frac{5517727}{46656}\right] \frac{1}{N_{f}}+\left[\frac{37377424567}{209952 N_{c}}-\frac{20021939}{23328 N_{c}^{3}}-\frac{223918424851 N_{c}}{3359232}\right] \frac{1}{N_{f}^{2}}+O\left(\epsilon ; \frac{1}{N_{f}^{3}}\right) \\
x_{9}= & -\frac{1}{144}+\left[\frac{343}{216 N_{c}}-\frac{6535889 N_{c}}{186624}\right] \frac{1}{N_{f}}+\left[\frac{388161667565}{3359232}-\frac{5239709503}{93312 N_{c}^{2}}-\frac{297237914233 N_{c}^{2}}{26873856}\right] \frac{1}{N_{f}^{2}}+O\left(\epsilon ; \frac{1}{N_{f}^{3}}\right) \\
x_{10}= & -\frac{17}{72}-\left[\frac{343}{432 N_{c}}+\frac{2137045 N_{c}}{46656}\right] \frac{1}{N_{f}}-\left[\frac{11234911345}{104976}+\frac{2318604883}{46656 N_{c}^{2}}+\frac{7914271411 N_{c}^{2}}{497664}\right] \frac{1}{N_{f}^{2}}+O\left(\epsilon ; \frac{1}{N_{f}^{3}}\right) \\
x_{11}= & -\frac{25}{18}-\left[\frac{115591}{432 N_{c}}+\frac{8299843 N_{c}}{93312}\right] \frac{1}{N_{f}}+\left[\frac{15724650809}{46656 N_{c}^{2}}-\frac{429314818345}{1679616}+\frac{261182511995 N_{c}^{2}}{6718464}\right] \frac{1}{N_{f}^{2}}+O\left(\epsilon ; \frac{1}{N_{f}^{3}}\right)
\end{aligned}
$$

where the double order symbol indicates both the two loop correction and the next order in the large $N_{f}$ expansion. These values of $x_{i}$ correspond to the $\epsilon$ expansion of all the critical couplings to the order which they are known in the previous section. Next the renormalization group functions for the wave function renormalization are evaluated at the Wilson-Fisher critical point and expanded in powers of both $\epsilon$ and $1 / N_{f}$. Subsequently the critical exponents should be in agreement with the coefficients of $\epsilon$ in the known large $N_{f}$ critical exponents of the non-Abelian Thirring universality class when they are expanded around $d=8-2 \epsilon$. Substituting the values from (5.3) into (4.5) we find for $S U\left(N_{c}\right)$ that 


$$
\begin{aligned}
\left.\gamma_{A}\left(g_{c}\right)\right|_{\alpha=0}= & \epsilon+\frac{245 N_{c}}{12 N_{f}} \epsilon+\frac{473585 N_{c}^{2}}{144 N_{f}^{2}} \epsilon+O\left(\epsilon^{2} ; \frac{1}{N_{f}^{3}}\right) \\
\left.\gamma_{c}\left(g_{c}\right)\right|_{\alpha=0}= & -\frac{245 N_{c}}{24 N_{f}} \epsilon-\frac{473585 N_{c}^{2}}{288 N_{f}^{2}} \epsilon+O\left(\epsilon^{2} ; \frac{1}{N_{f}^{3}}\right) \\
\left.\gamma_{\psi}\left(g_{c}\right)\right|_{\alpha=0}= & {\left[\frac{245 N_{c}}{12}-\frac{245}{12 N_{c}}\right] \frac{\epsilon}{N_{f}} } \\
& +\left[\frac{473585 N_{c}^{2}}{144}-\frac{473585}{144}\right] \frac{\epsilon}{N_{f}^{2}}+O\left(\epsilon^{2} ; \frac{1}{N_{f}^{3}}\right)
\end{aligned}
$$

where $g_{c}$ denotes the set of critical couplings defined in (5.2). In order to compare with the large $N_{f}$ critical exponents of the universal theory founded on the nonAbelian Thirring model at the Wilson-Fisher fixed point, we have to restrict the exponents to the Landau gauge. This is because in effect the gauge parameter $\alpha$ acts as an additional coupling constant and the Landau gauge is the corresponding fixed point in this context. In other words the gauge dependent large $N_{f}$ critical exponents of the gluon, quark and ghost fields can only be compared with the Landau gauge anomalous dimensions at criticality which has been noted before in $[16,18]$. We restrict our large $N_{f}$ comparison to these three anomalous dimensions since they are the only three quantities which are available for eight dimensional QCD. While the large $N_{f}$ critical exponent of the four dimensional QCD $\beta$-function is known at $O\left(1 / N_{f}\right)$ [16], that exponent would relate to the renormalization of the operator $\frac{1}{4} G_{\mu \nu}^{a} G^{a \mu \nu}$ in (2.12). In four dimensions the gauge coupling constant in four dimensional QCD is dimensionless but in the continuation along the thread of the $d$-dimensional Wilson-Fisher fixed point the coupling becomes dimensionful and the correction to scaling exponent in four dimensions transcends into a mass parameter in higher dimensions such as the eight dimensional Lagrangian (2.12). Therefore, if we evaluate the leading order $d$-dimensional large $N_{f}$ critical exponents for the gluon, quark and ghost fields of [52] near eight dimensions by setting $d=8-2 \epsilon$ we find that the coefficients of $\epsilon$ match precisely with those of (5.4) in the Landau gauge for $S U\left(N_{c}\right)$. Moreover, since the quark anomalous dimension is also known at $O\left(1 / N_{f}^{2}\right)$ in the Landau gauge [18], it is satisfying to record that the corresponding term of $\left.\gamma_{\psi}\left(g_{c}\right)\right|_{\alpha=0}$ is in full agreement. While we have not given explicit details for the $S U(2)$ and $S U(3)$ renormalization group functions, we note that we have carried out the same check as $S U\left(N_{c}\right)$ and found that there is full consistency in these cases too. Consequently the ultraviolet completion of QCD or the non-Abelian Thirring model to eight dimensions via (2.8) has been established at one loop within the large $N_{f}$ expansion as expected.

\section{DIMENSION 8 OPERATORS IN FOUR DIMENSIONS}

In this section we turn to a complementary problem which is the renormalization of dimension 8 operators in four dimensions. Such operators in the case of Yang-Mills theory have been considered in [31,32] where, for instance, the anomalous dimensions for the $S U(2)$ and $S U(3)$ groups were computed at one loop in [31]. The reason for this is that in four dimensions the canonical dimensions of the gluon and ghost fields are such that there is a complicated mixing between gluonic and quark operators. In (2.8) by contrast on dimensional grounds it is not possible to have any other interactions involving quarks aside from the quark-gluon interaction. Therefore in this section we concentrate on the renormalization of four dimensional dimension 8 operators in $S U\left(N_{c}\right)$ Yang-Mills theory for $N_{c} \geq 4$ as this case has not been considered. In addition we use the same operator basis as was used in (2.8), which differs from that of [31,32], in order to ease structural comparisons. First, to set notation the basis for the dimension 8 operators in four dimensions for the color group $S U\left(N_{c}\right)$ we use is

$$
\begin{aligned}
\mathcal{O}_{841} & =G_{\mu \sigma}^{a} G^{a \mu \rho} G^{b \sigma \nu} G_{\rho \nu}^{b}, \quad \mathcal{O}_{842}=G_{\mu \sigma}^{a} G^{b \mu \rho} G^{b \sigma \nu} G_{\rho \nu}^{a} \\
\mathcal{O}_{843} & =G_{\mu \sigma}^{a} G_{\nu \rho}^{a} G^{b \sigma \mu} G^{b \rho \nu}, \quad \mathcal{O}_{844}=G_{\mu \sigma}^{a} G_{\nu \rho}^{b} G^{a \sigma \mu} G^{b \rho \nu} \\
\mathcal{O}_{845} & =d_{4}^{a b c d} G_{\mu \sigma}^{a} G^{b \mu \sigma} G_{\nu \rho}^{c} G^{d \nu \rho}, \\
\mathcal{O}_{846} & =d_{4}^{a b c d} G_{\mu \sigma}^{a} G^{c \mu \rho} G^{b \nu \sigma} G_{\nu \rho}^{d} \\
\mathcal{O}_{847} & =d_{4}^{a c b d} G_{\mu \sigma}^{a} G^{b \mu \sigma} G_{\nu \rho}^{c} G^{d \nu \rho}, \\
\mathcal{O}_{848} & =d_{4}^{a d b c} G_{\mu \sigma}^{a} G^{c \mu \rho} G^{b \nu \sigma} G_{\nu \rho}^{d} .
\end{aligned}
$$

The notation is similar to that used in [31]. However, these operators are not the same since we have specified the basis with respect to a specific color group unlike [31]. We have chosen this ordering so that the $S U(2)$ basis corresponds to the first four operators and that for $S U(3)$ involves the first six. Equally the ordering is equivalent to that used in (2.8) for the quartic gluon interactions with coupling constants $g_{4}$ to $g_{11}$ respectively.

To renormalize the operators $\mathcal{O}_{84 i}$ we use the same technique as that for the 4-point functions of (2.8) but in this case we apply it to the Green's function $\left\langle A_{\mu}^{a}\left(p_{1}\right) A_{\nu}^{b}\left(p_{2}\right) A_{\sigma}^{c}\left(p_{3}\right) A_{\rho}^{d}\left(p_{4}\right) \mathcal{O}_{84 i}\left(p_{5}\right)\right\rangle \quad$ where $\quad p_{5}=$ $-\sum_{i=1}^{4} p_{i}$. However, as we are considering an operator renormalization there will be a mixing of the $\mathcal{O}_{84 i}$ operators among themselves which will produce a mixing matrix of anomalous dimensions. This is similar to the $\beta$-functions for the couplings in (2.8). However for operator renormalization there are aspects to address compared with a Lagrangian renormalization. For instance, for the gauge invariant dimension 8 operators (6.1) there will be mixing into gauge variant and equation of motion operators as well as possibly total derivative operators. The latter can arise 
when an operator is renormalized in a Green's function where the insertion is at nonzero momentum insertion. Moreover this set includes total derivative operators which are gauge invariant, gauge variant and equation of motion operators. So the mixing matrix in effect is larger than an $8 \times 8$ matrix based on (6.1). Not only do the operators of (6.1) mix with all operators of the enlarged set but the gauge variant, equation of motion and total derivative operators can mix with themselves when each is renormalized. However, the overall mixing matrix has a particular structure in that the gauge invariant operators mix with all classes of operators but the gauge variant ones only mix within that class. See, for instance, [53-56]. As we are primarily interested in the gauge invariant operators we restrict the evaluation of the Green's function $\left\langle A_{\mu}^{a}\left(p_{1}\right) A_{\nu}^{b}\left(p_{2}\right) A_{\sigma}^{c}\left(p_{3}\right) A_{\rho}^{d}\left(p_{4}\right) \mathcal{O}_{84 i}\left(p_{5}\right)\right\rangle$ to the case where the external gluon legs are all on-shell. The condition for a gluon $A_{\mu}^{a}(p)$ to be on-shell is that its polarization vector and momentum satisfy

$$
p_{\mu} p^{\mu}=0, \quad p^{\mu} \epsilon_{\mu}(p)=0 .
$$

Therefore we multiply the Green's function by $\epsilon^{\mu}\left(p_{1}\right) \epsilon^{\nu}\left(p_{2}\right) \epsilon^{\sigma}\left(p_{3}\right) \epsilon^{\rho}\left(p_{4}\right)$ and apply (6.2). The terms which remain such as $\epsilon_{\mu}\left(p_{i}\right) p_{j}^{\mu}$ for $i \neq j$ or $p_{i} p_{j}$ are resolved by grouping them in terms corresponding to the Feynman rules of the contributing operators such as (6.1) and any gauge invariant total derivative or equation of motion operators. The reason why this list omits gauge variant operators is that the restriction of (6.2) corresponds to taking a physical matrix element. As such no gauge variant operators can be present [53-56].

Necessary to achieve the resolution into this basis of operators is that the operator has to be inserted at nonzero momentum. If it was inserted at zero momentum then certain terms of the Feynman rule of different operators will be similar and hence the extraction of the renormalization constants in the mixing matrix cannot be achieved uniquely and unambiguously. Therefore, formally the set of bare operators, denoted by the subscript o satisfy

$$
\mathcal{O}_{i \mathrm{o}}=Z_{i j} \mathcal{O}_{j}
$$

where $Z_{i j}$ is the mixing matrix of renormalization constants from which the mixing matrix of anomalous dimensions, $\gamma_{i j}(a)$, can be deduced. In this section $a=g^{2} /\left(16 \pi^{2}\right)$ denotes the coupling constant of four dimensional QCD where $g$ is the coupling present in the covariant derivative. It transpires that for the eight operators (6.1) the matrix needs to be enlarged since there is mixing into an equation of motion operator. In [31] the seven independent equation of motion operators were constructed and are

$$
\begin{aligned}
& \mathcal{O}_{82 e 1}=D^{\mu} G_{\mu \nu}^{a} D^{\rho} D_{\sigma} D_{\rho} G^{a \nu \sigma}, \\
& \mathcal{O}_{82 e 2}=D^{\sigma} D^{\mu} G_{\mu \nu}^{a} D^{\rho} D^{\nu} G_{\sigma \rho}^{a} \\
& \mathcal{O}_{82 e 3}=D^{\sigma} D^{\mu} G_{\mu \nu}^{a} D_{\rho} D_{\sigma} G^{a \nu \rho}, \\
& \mathcal{O}_{82 e 4}=D_{\sigma} G_{\nu \rho}^{a} D^{\sigma} D^{\rho} D_{\mu} G^{a \mu \nu} \\
& \mathcal{O}_{82 e 5}=G_{\nu \sigma}^{a} D^{\sigma} D^{\rho} D_{\rho} D_{\mu} G^{a \mu \nu} \\
& \mathcal{O}_{83 e 1}=f^{a b c} G_{\sigma \rho}^{a} D^{\nu} G^{b \sigma \rho} D^{\mu} G_{\mu \nu}^{c}, \\
& \mathcal{O}_{83 e 2}=f^{a b c} G_{\sigma}^{a \nu} G^{b \sigma \rho} D_{\rho} D^{\mu} G_{\mu \nu}^{c}
\end{aligned}
$$

where the first two labels indicate the operator dimension and gluon leg number respectively and note that each operator is gauge invariant. We recall that in four dimensions the equation of motion of the gluon in Yang-Mills theory is

$$
D^{\mu} G_{\mu \nu}=0
$$

which is relatively simple in contrast to that of (2.8). Unlike (6.1) there is no reduction of the equation of motion set (6.4) depending on which color group we consider. One comment is in order with respect to (2.8) which is that the operators (6.4) are not present in that Lagrangian. The reason why they are considered part of the basis here arises from the different nature of the two types of renormalizations we are carrying out. In (2.8) for the purely gluonic sector we included the set of independent gauge invariant operators involving the field strength. The operators which were dependent, and hence not included, were equivalent to linear combinations of the ones appearing in (2.8) as well as operators which were total derivatives. In a Lagrangian context the latter operators can be integrated out and hence were not included in (2.8). For the renormalization of the dimension 8 operators (6.1) in four dimensions one has to accommodate mixing into the various operator classes noted earlier. As one of these classes involves equation of motion operators we have included these in the set of operators for our mixing. However it is a straightforward exercise to show that the operators $\mathcal{O}_{82 e i}$ can each be related to the gluon kinetic operator plus higher leg operators and those with a total derivative. Equally the operators $\mathcal{O}_{83 e i}$ in eight dimensions can be mapped to the operators with couplings $g_{2}$ and $g_{3}$ respectively plus higher leg and total derivative operators in (2.8).

The final stage of the operator renormalization is the evaluation of the divergent part of the on-shell Green's function. Like the renormalization of the 4-point functions of (2.8) we apply the vacuum bubble expansion based on (3.5). The only major difference between its use here and the previous application is that after the expansion and the Laporta reduction the master integral is evaluated in four dimensions. Therefore, extracting the renormalization constants we find the elements of the mixing matrix are 


$$
\begin{aligned}
& \gamma_{841,841}(a)=\frac{8}{3 N_{c}} a+O\left(a^{2}\right), \quad \gamma_{841,842}(a)=-\frac{8}{3 N_{c}} a+O\left(a^{2}\right) \\
& \gamma_{841,843}(a)=\frac{22}{3 N_{c}} a+O\left(a^{2}\right), \quad \gamma_{841,844}(a)=-\frac{1}{6 N_{c}}\left[11 N_{c}^{2}+44\right] a+O\left(a^{2}\right) \\
& \gamma_{841,845}(a)=-\frac{11}{3} a+O\left(a^{2}\right), \quad \gamma_{841,846}(a)=\frac{4}{3} a+O\left(a^{2}\right) \\
& \gamma_{841,847}(a)=\frac{11}{3} a+O\left(a^{2}\right), \quad \gamma_{841,848}(a)=-\frac{4}{3} a+O\left(a^{2}\right) \\
& \gamma_{842,841}(a)=-\frac{1}{3 N_{c}}\left[14 N_{c}^{2}+4\right] a+O\left(a^{2}\right), \quad \gamma_{842,842}(a)=-\frac{1}{3 N_{c}}\left[10 N_{c}^{2}-4\right] a+O\left(a^{2}\right) \\
& \gamma_{842,843}(a)=\frac{1}{3 N_{c}}\left[12 N_{c}^{2}+22\right] a+O\left(a^{2}\right), \quad \gamma_{842,844}(a)=-\frac{1}{6 N_{c}}\left[-N_{c}^{2}+44\right] a+O\left(a^{2}\right) \\
& \gamma_{842,845}(a)=-\frac{11}{3} a+O\left(a^{2}\right), \quad \gamma_{842,846}(a)=-\frac{2}{3} a+O\left(a^{2}\right) \\
& \gamma_{842,847}(a)=\frac{11}{3} a+O\left(a^{2}\right), \quad \gamma_{842,848}(a)=\frac{2}{3} a+O\left(a^{2}\right) \\
& \gamma_{843,841}(a)=-\frac{1}{3 N_{c}}\left[28 N_{c}^{2}+68\right] a+O\left(a^{2}\right), \quad \gamma_{843,842}(a)=-\frac{1}{3 N_{c}}\left[-24 N_{c}^{2}-68\right] a+O\left(a^{2}\right) \\
& \gamma_{843,843}(a)=\frac{1}{3 N_{c}}\left[2 N_{c}^{2}+50\right] a+O\left(a^{2}\right), \quad \gamma_{843,844}(a)=-\frac{1}{3 N_{c}}\left[-N_{c}^{2}+50\right] a+O\left(a^{2}\right) \\
& \gamma_{843,845}(a)=-\frac{25}{3} a+O\left(a^{2}\right), \quad \gamma_{843,846}(a)=-\frac{34}{3} a+O\left(a^{2}\right) \\
& \gamma_{843,847}(a)=\frac{25}{3} a+O\left(a^{2}\right), \quad \gamma_{843,848}(a)=\frac{34}{3} a+O\left(a^{2}\right) \\
& \gamma_{844,841}(a)=-\frac{56}{N_{c}} a+O\left(a^{2}\right), \quad \gamma_{844,842}(a)=\frac{56}{N_{c}} a+O\left(a^{2}\right) \\
& \gamma_{844,843}(a)=-\frac{4}{N_{c}} a+O\left(a^{2}\right), \quad \gamma_{844,844}(a)=-\frac{1}{3 N_{c}}\left[22 N_{c}^{2}-12\right] a+O\left(a^{2}\right) \\
& \gamma_{844,845}(a)=2 a+O\left(a^{2}\right), \quad \gamma_{844,846}(a)=-28 a+O\left(a^{2}\right) \\
& \gamma_{844,847}(a)=-2 a+O\left(a^{2}\right), \quad \gamma_{844,848}(a)=28 a+O\left(a^{2}\right) \\
& \gamma_{845,841}(a)=-\frac{1}{N_{c}^{2}}\left[28 N_{c}^{2}-112\right] a+O\left(a^{2}\right), \quad \gamma_{845,842}(a)=\frac{1}{N_{c}^{2}}\left[28 N_{c}^{2}-112\right] a+O\left(a^{2}\right) \\
& \gamma_{845,843}(a)=-\frac{1}{N_{c}^{2}}\left[2 N_{c}^{2}-8\right] a+O\left(a^{2}\right), \quad \gamma_{845,844}(a)=-\frac{1}{N_{c}^{2}}\left[-2 N_{c}^{2}+8\right] a+O\left(a^{2}\right) \\
& \gamma_{845,845}(a)=-\frac{1}{2 N_{c}}\left[5 N_{c}^{2}+8\right] a+O\left(a^{2}\right), \quad \gamma_{845,846}(a)=-\frac{1}{N_{c}}\left[6 N_{c}^{2}-56\right] a+O\left(a^{2}\right) \\
& \gamma_{845,847}(a)=-\frac{1}{3 N_{c}}\left[2 N_{c}^{2}-12\right] a+O\left(a^{2}\right), \quad \gamma_{845,848}(a)=-\frac{1}{3 N_{c}}\left[-16 N_{c}^{2}+168\right] a+O\left(a^{2}\right) \\
& \gamma_{846,841}(a)=-\frac{1}{3 N_{c}^{2}}\left[-4 N_{c}^{2}+16\right] a+O\left(a^{2}\right), \quad \gamma_{846,842}(a)=-\frac{1}{3 N_{c}^{2}}\left[4 N_{c}^{2}-16\right] a+O\left(a^{2}\right) \\
& \gamma_{846,843}(a)=-\frac{1}{3 N_{c}^{2}}\left[-11 N_{c}^{2}+44\right] a+O\left(a^{2}\right), \quad \gamma_{846,844}(a)=-\frac{1}{3 N_{c}^{2}}\left[11 N_{c}^{2}-44\right] a+O\left(a^{2}\right) \\
& \gamma_{846,845}(a)=-\frac{1}{3 N_{c}}\left[4 N_{c}^{2}-22\right] a+O\left(a^{2}\right), \quad \gamma_{846,846}(a)=-\frac{1}{3 N_{c}}\left[3 N_{c}^{2}+8\right] a+O\left(a^{2}\right)
\end{aligned}
$$




$$
\begin{aligned}
& \gamma_{846,847}(a)=-\frac{1}{3 N_{c}}\left[-3 N_{c}^{2}+22\right] a+O\left(a^{2}\right), \quad \gamma_{846,848}(a)=-\frac{1}{3 N_{c}}\left[3 N_{c}^{2}-8\right] a+O\left(a^{2}\right) \\
& \gamma_{847,841}(a)=-\frac{1}{3 N_{c}^{2}}\left[34 N_{c}^{2}-136\right] a+O\left(a^{2}\right) \\
& \gamma_{847,842}(a)=-\frac{1}{3 N_{c}^{2}}\left[-34 N_{c}^{2}+136\right] a+O\left(a^{2}\right) \\
& \gamma_{847,843}(a)=-\frac{1}{3 N_{c}^{2}}\left[-25 N_{c}^{2}+100\right] a+O\left(a^{2}\right) \\
& \gamma_{847,844}(a)=-\frac{1}{3 N_{c}^{2}}\left[25 N_{c}^{2}-100\right] a+O\left(a^{2}\right) \\
& \gamma_{847,845}(a)=-\frac{1}{12 N_{c}}\left[25 N_{c}^{2}-200\right] a+O\left(a^{2}\right) \\
& \gamma_{847,846}(a)=-\frac{1}{3 N_{c}}\left[19 N_{c}^{2}-68\right] a+O\left(a^{2}\right) \\
& \gamma_{847,847}(a)=-\frac{1}{3 N_{c}}\left[-3 N_{c}^{2}+50\right] a+O\left(a^{2}\right), \\
& \gamma_{847,848}(a)=-\frac{1}{3 N_{c}}\left[-16 N_{c}^{2}+68\right] a+O\left(a^{2}\right) \\
& \begin{array}{l}
\gamma_{848,841}(a)=-\frac{1}{3 N_{c}^{2}}\left[2 N_{c}^{2}-8\right] a+O\left(a^{2}\right), \\
\gamma_{848,843}(a)=-\frac{1}{3 N_{c}^{2}}\left[-11 N_{c}^{2}+44\right] a+O\left(a^{2}\right)
\end{array} \\
& \gamma_{848,844}(a)=-\frac{1}{3 N_{c}^{2}}\left[11 N_{c}^{2}-44\right] a+O\left(a^{2}\right) \\
& \gamma_{848,845}(a)=-\frac{1}{6 N_{c}}\left[5 N_{c}^{2}-44\right] a+O\left(a^{2}\right), \quad \gamma_{848,846}(a)=-\frac{1}{3 N_{c}}\left[8 N_{c}^{2}-4\right] a+O\left(a^{2}\right) \\
& \gamma_{848,847}(a)=-\frac{1}{3 N_{c}}\left[-6 N_{c}^{2}+22\right] a+O\left(a^{2}\right) \\
& \gamma_{848,848}(a)=-\frac{1}{3 N_{c}}\left[4 N_{c}^{2}+4\right] a+O\left(a^{2}\right)
\end{aligned}
$$

for $S U\left(N_{c}\right)$. For the eight $S U\left(N_{c}\right)$ dimension 8 core operators at one loop there is mixing into only one equation of motion operator which is $\mathcal{O}_{83 e 2}$. More explicitly we have

$$
\begin{aligned}
& \gamma_{841,83 e 2}(a)=-2 a+O\left(a^{2}\right), \quad \gamma_{842,83 e 2}(a)=4 a+O\left(a^{2}\right) \\
& \gamma_{843,83 e 2}(a)=4 a+O\left(a^{2}\right) \quad \gamma_{844,83 e 2}(a)=-8 a+O\left(a^{2}\right) \\
& \gamma_{845,83 e 2}(a)=-\frac{4}{N_{c}}\left[N_{c}^{2}-4\right] a+O\left(a^{2}\right) \\
& \gamma_{846,83 e 2}(a)=-\frac{1}{N_{c}}\left[N_{c}^{2}-4\right] a+O\left(a^{2}\right), \\
& \gamma_{847,83 e 2}(a)=\frac{2}{N_{c}}\left[N_{c}^{2}-4\right] a+O\left(a^{2}\right) \\
& \gamma_{848,83 e 2}(a)=\frac{2}{N_{c}}\left[N_{c}^{2}-4\right] a+O\left(a^{2}\right) .
\end{aligned}
$$

The mixing of the main operators into this specific equation of motion operator is necessary as otherwise divergences would remain in each of the Green's functions. In other words there are not sufficient counterterms and freedom available from the set of operators in (6.1) alone to obtain a finite expression. For $S U(2)$ and $S U(3)$ the respective parts for this sector of the mixing matrix are contained within (6.7). For $S U(2)$ only the first four operators of (6.1) are active and for $S U(3)$ it is the first six. Then for $S U(2)$ the first four entries in (6.7) correspond to the 4-leg operator mixing into the equation of motion operators. Clearly $\gamma_{845,83 e 2}(a)$ vanishes for $N_{c}=2$ as a consistency check. The situation for $S U(3)$ is similar except the first six entries are relevant but $N_{c}=3$ has to be set. Finally, the equation of motion operators can mix with themselves and we have determined that sector of the mixing matrix in the same way by inserting each operator in the physical matrix element. The only nonzero entries are

$$
\begin{aligned}
& \gamma_{83 e 1,82 e 4}(a)=-\frac{1}{3 N_{c}} a+O\left(a^{2}\right), \\
& \gamma_{83 e 1,82 e 5}(a)=\frac{1}{2 N_{c}} a+O\left(a^{2}\right)
\end{aligned}
$$

which is valid for all the $S U\left(N_{c}\right)$ groups. This completes our dimension 8 operator analysis in four dimensions for the particular $S U\left(N_{c}\right)$ color groups. These results together 
with the $S U(2)$ and $S U(3)$ cases are all included in the Supplemental Material. While this is a fully separate computation to the renormalization of (2.8) the structural parallels of the respective renormalization group functions are now evident.

\section{DISCUSSION}

One of our main goals was to construct the eight dimensional quantum field theory which was in the same universality class as the two dimensional non-Abelian Thirring model and four dimensional QCD at their respective Wilson-Fisher fixed points. We have managed to achieve this by following the guiding principles established for the parallel construction for scalar field theories with an $O(N)$ symmetry. The first of these is to retain the core interaction between the matter and force fields which in the present case were a spin- $-\frac{1}{2}$ fermion and spin- 1 boson field in the adjoint representation of the color group. This interaction is the only one present in the base theory of the tower of theories lying in the universality class which is the nonAbelian Thirring model [21]. The second aspect is renormalizability. This means that extra interactions have to be included in the critical dimension of each of the subsequent Lagrangians of the tower so that each Lagrangian is renormalizable. These extra independent operators, which are purely gluonic for this universality class, will become irrelevant or relevant away from the critical dimension. So for example including the canonical gluon kinetic operator for QCD in the non-Abelian Thirring model would render it nonrenormalizable in two dimensions. The final main principle is the requirement of gauge fixing. We chose a linear covariant gauge fixing in order to make connections with lower dimensional results and extended the FaddeevPopov construction to eight dimensions. This last step is necessary as the two dimensional non-Abelian Thirring model has a conserved current, $\bar{\psi} \gamma^{\mu} T^{a} \psi$, whose 2-point correlation function is transverse. While there is no gluon as such in the non-Abelian Thirring model, like the four dimensional gauge theory case, the field $A_{\mu}^{a}$ is an auxiliary in two dimensions and corresponds to this current. In other words the correlation of $A_{\mu}^{a}$ in two dimensions is in effect akin to a Landau gauge propagator. As the gauge parameter, $\alpha$, in QCD is effectively a second coupling constant then at criticality one has to effect its critical coupling which corresponds in fact to the Landau gauge. This accords with the establishment of (2.8) as being in the same universality class as the non-Abelian Thirring model and QCD via the large $N_{f}$ expansion. One can only compare the $d$-dimensional large $N_{f}$ critical exponents with the exponents derived from gauge dependent renormalization group functions when the $\epsilon$ expansion of the latter have been computed in the Landau gauge. We have checked this off explicitly here for eight dimensional QCD from the one loop renormalization group functions.
Put another way the Wilson-Fisher fixed point underlying this particular universality class preserves the transversality of the gluon across the dimensions.

There are several future avenues to pursue in light of our analysis. One is to build the ten dimensional theory of a spin-1 field coupled to a fundamental fermion which lies in the non-Abelian Thirring model universality class. The procedure to do this evidently follows the above outline. It would have no technical obstacles aside from the calculational one of requiring a large amount of integration by parts to determine even just the one loop renormalization group functions. This will be a tedious exercise rather than an insurmountable problem. Another obvious extension is to construct the renormalization group functions of (2.8) at two loops. Indeed this has already been achieved for QED $[26,27]$. However in eight dimensions the computations were manageable due to there being only four independent interactions and more crucially no quintic or sextic gauge interactions. These were obviously present in the nonAbelian case and also increased the amount of integration needed in order to evaluate the large number of Feynman graphs with high exponent gluon propagators [26]. With the tower of Lagrangians essentially established at the Wilson-Fisher fixed point for the non-Abelian Thirring model universality class, the next focus ought to be on the connection of non-Lagrangian operators in the universal theory. These operators will have massive couplings in the noncritical dimensions but are relevant in constructing effective field Lagrangians in a specific dimension. In other words there should be a drive to study the operator anomalous dimensions at criticality.

We have taken the first step in this direction by renormalizing dimension 8 operators in four dimensions. While laying the foundation to this here by illustrating the structural parallels of the renormalization group functions, the next step is to introduce quark contributions. These are required for the large $N_{f}$ expansion connection where the underlying operator critical exponents in the universal theory would also need to be found in addition to the mixing matrices in perturbation theory. The perturbative computations to construct such mixing matrices should not be regarded as a straightforward task. One reason for this is due to the canonical dimensions of the quark and gluon fields being different in $d$-dimensions. Hence quark and gluon operators will have different canonical dimensions except in one particular dimension. Therefore we did not have to consider what would ordinarily be dimension 8 quark operators in the four dimensional sense in the construction of the eight dimensional Lagrangian (2.8). However, in four dimensional QCD there are dimension 8 operators with quark content in addition to the gluon operators of (6.1). This was one of the reasons why our focus was on Yang-Mills operators here as an exploratory exercise in the context of (2.8) and to observe that the structure of the respective four and eight dimensional 
renormalization group functions were not dissimilar. While (2.8) has a quark operator, it is the kinetic term and it does not have the same canonical dimension as, say, the operators of (6.1) in four dimensions. The first stage in such an investigation will be to set up the large $N_{f}$ formalism for dimension 6 and 8 gauge invariant operators and compute the mixing matrix of critical exponents at $O\left(1 / N_{f}\right)$ in $d$ dimensions. The former dimension is required for an analysis of (2.2) and we note that the large $N_{f}$ exponent relating to the QCD $\beta$-function in four dimensions [16] was derived from the critical point large $N_{f}$ renormalization of the dimension four operator $G_{\mu \nu}^{a} G^{a \mu \nu}$. That in effect was the initial step of the proposal to examine the operator content of the tower of Lagrangians constituting universal non-Abelian Thirring model universality class.

\section{ACKNOWLEDGMENTS}

This work was carried out with the support of the STFC through the Consolidated Grant No. ST/L000431/1. The author thanks K. L. Jones, R. M. Simms and Dr. Marco Bochicchio for useful discussions.
[1] D. J. Gross and F. J. Wilczek, Phys. Rev. Lett. 30, 1343 (1973).

[2] H. D. Politzer, Phys. Rev. Lett. 30, 1346 (1973).

[3] W. E. Caswell, Phys. Rev. Lett. 33, 244 (1974).

[4] D. R. T. Jones, Nucl. Phys. B75, 531 (1974).

[5] O. V. Tarasov, A. A. Vladimirov, and A. Yu. Zharkov, Phys. Lett. 93B, 429 (1980).

[6] T. van Ritbergen, J. A. M. Vermaseren, and S. A. Larin, Phys. Lett. B 400, 379 (1997).

[7] M. Czakon, Nucl. Phys. B710, 485 (2005).

[8] P. A. Baikov, K. G. Chetyrkin, and J. H. Kühn, J. High Energy Phys. 10 (2014), 76.

[9] P. A. Baikov, K. G. Chetyrkin, and J. H. Kühn, Phys. Rev. Lett. 118, 082002 (2017).

[10] F. Herzog, B. Ruijl, T. Ueda, J. A. M. Vermaseren, and A. Vogt, J. High Energy Phys. 02 (2017) 090.

[11] T. Luthe, A. Maier, P. Marquard, and Y. Schröder, J. High Energy Phys. 01 (2017) 081.

[12] T. Luthe, A. Maier, P. Marquard, and Y. Schröder, J. High Energy Phys. 03 (2017) 020.

[13] P. A. Baikov, K. G. Chetyrkin, and J.H. Kühn, J. High Energy Phys. 04 (2017) 119.

[14] T. Luthe, A. Maier, P. Marquard, and Y. Schröder, J. High Energy Phys. 10 (2017) 166.

[15] K. G. Chetyrkin, G. Falcioni, F. Herzog, and J. A. M. Vermaseren, J. High Energy Phys. 10 (2017) 179.

[16] J. A. Gracey, Phys. Lett. B 373, 178 (1996).

[17] A. Palanques-Mestre and P. Pascual, Commun. Math. Phys. 95, 277 (1984).

[18] M. Ciuchini, S.É. Derkachov, J. A. Gracey, and A. N. Manashov, Nucl. Phys. B579, 56 (2000).

[19] A. N. Vasil'ev, Y. M. Pismak, and J. R. Honkonen, Theor. Math. Phys. 46, 104 (1981).

[20] A. N. Vasil'ev, Y. M. Pismak, and J. R. Honkonen, Theor. Math. Phys. 47, 465 (1981).

[21] A. Hasenfratz and P. Hasenfratz, Phys. Lett. B 297, 166 (1992).

[22] L. Fei, S. Giombi, and I. R. Klebanov, Phys. Rev. D 90, 025018 (2014).

[23] L. Fei, S. Giombi, I. R. Klebanov, and G. Tarnopolsky, Phys. Rev. D 91, 045011 (2015).
[24] J. A. Gracey, Phys. Rev. D 92, 025012 (2015).

[25] J. A. Gracey and R. M. Simms, Phys. Rev. D 96, 025022 (2017).

[26] J. A. Gracey, Phys. Rev. D 93, 025025 (2016).

[27] S. Giombi, I. R. Klebanov, and G. Tarnopolsky, J. Phys. A 49, 135403 (2016).

[28] A. J. McKane, D. J. Wallace, and R. K. P. Zia, Phys. Lett. B 65, 171 (1976).

[29] A. J. McKane, J. Phys. G 3, 1165 (1977).

[30] D. I. Kazakov, J. High Energy Phys. 03 (2002) 020.

[31] J. A. Gracey, Nucl. Phys. B634, 192 (2002); B696, 295 (2004).

[32] A. Yu. Morozov, Yad. Fiz. 40, 788 (1984) [Sov. J. Nucl. Phys. 40, 505 (1984)].

[33] A. J. Macfarlane, A. Sudbery, and P. H. Weisz, Commun. Math. Phys. 11, 77 (1968).

[34] M. Stingl, Phys. Rev. D 34, 3863 (1986); 36, 651(E) (1987).

[35] P. Nogueira, J. Comput. Phys. 105, 279 (1993).

[36] S. A. Larin and J. A. M. Vermaseren, Phys. Lett. B 303, 334 (1993).

[37] J. A. M. Vermaseren, arXiv:math-ph/0010025.

[38] M. Tentyukov and J. A. M. Vermaseren, Comput. Phys. Commun. 181, 1419 (2010).

[39] S. Laporta, Int. J. Mod. Phys. A 15, 5087 (2000).

[40] C. Studerus, Comput. Phys. Commun. 181, 1293 (2010).

[41] A. von Manteuffel and C. Studerus, arXiv:1201.4330.

[42] O. V. Tarasov, Phys. Rev. D 54, 6479 (1996).

[43] O. V. Tarasov, Nucl. Phys. B502, 455 (1997).

[44] P. A. Baikov and K. G. Chetyrkin, Nucl. Phys. B837, 186 (2010).

[45] N. I. Ussyukina and A. I. Davydychev, Yad. Fiz. 56, 172 (1993) [Phys. At. Nucl. 56, 1553 (1993)].

[46] N. I. Ussyukina and A. I. Davydychev, Phys. Lett. B 332, 159 (1994).

[47] N. I. Ussyukina and A. I. Davydychev, Phys. Lett. B 305, 136 (1993).

[48] M. Misiak and M. Münz, Phys. Lett. B 344, 308 (1995).

[49] K. G. Chetyrkin, M. Misiak, and M. Münz, Nucl. Phys. B518, 473 (1998). 
[50] S. Weinberg, Phys. Rev. 118, 838 (1960).

[51] See Supplemental Material at http://link.aps.org/ supplemental/10.1103/PhysRevD.97.025009 for the electronic version of renormalization group functions for eight dimensional QCD and dimension 8 operator anomalous dimensions in four dimensional QCD.
[52] J. A. Gracey, Phys. Lett. B 318, 177 (1993).

[53] S. D. Joglekar and B. W. Lee, Ann. Phys. (N.Y.) 97, 160 (1976).

[54] S. D. Joglekar, Ann. Phys. (N.Y.) 100, 395 (1976).

[55] S. D. Joglekar, Ann. Phys. (N.Y.) 108, 233 (1977).

[56] S. D. Joglekar, Ann. Phys. (N.Y.) 109, 210 (1977). 